\title{
Disruption of RAB-5 Increases EFF-1 Fusogen Availability at the Cell Surface and Promotes the Regenerative Axonal Fusion Capacity of the Neuron
}

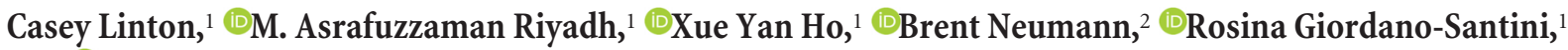 \\ and $\odot$ Massimo A. Hilliard ${ }^{1}$ \\ ${ }^{1}$ Clem Jones Centre for Ageing Dementia Research, Queensland Brain Institute, The University of Queensland, Brisbane, Queensland 4072, Australia, and \\ ${ }^{2}$ Neuroscience Program, Monash Biomedicine Discovery Institute and Department of Anatomy and Developmental Biology, Monash University, \\ Melbourne, Victoria 3800, Australia
}

Following a transection injury to the axon, neurons from a number of species have the ability to undergo spontaneous repair via fusion of the two separated axonal fragments. In the nematode Caenorhabditis elegans, this highly efficient regenerative axonal fusion is mediated by epithelial fusion failure-1 (EFF-1), a fusogenic protein that functions at the membrane to merge the two axonal fragments. Identifying modulators of axonal fusion and EFF-1 is an important step toward a better understanding of this repair process. Here, we present evidence that the small GTPase RAB-5 acts to inhibit axonal fusion, a function achieved via endocytosis of EFF-1 within the injured neuron. Therefore, we find that perturbing RAB- 5 activity is sufficient to restore axonal fusion in mutant animals with decreased axonal fusion capacity. This is accompanied by enhanced membranous localization of EFF-1 and the production of extracellular EFF-1containing vesicles. These findings identify RAB-5 as a novel regulator of axonal fusion in $C$. elegans hermaphrodites and the first regulator of EFF-1 in neurons.

Key words: axonal fusion; axonal regeneration; C. elegans; endocytosis; fusogen; RAB-5

Significance Statement

Peripheral and central nerve injuries cause life-long disabilities due to the fact that repair rarely leads to reinnervation of the target tissue. In the nematode Caenorhabditis elegans, axonal regeneration can proceed through axonal fusion, whereby a regrowing axon reconnects and fuses with its own separated distal fragment, restoring the original axonal tract. We have characterized axonal fusion and established that the fusogen epithelial fusion failure-1 (EFF-1) is a key element for fusing the two separated axonal fragments back together. Here, we show that the small GTPase RAB-5 is a key cell-intrinsic regulator of the fusogen EFF-1 and can in turn regulate axonal fusion. Our findings expand the possibility for this process to be controlled and exploited to facilitate axonal repair in medical applications.

\section{Introduction}

Treating nerve injuries is of great interest in the clinical setting. Significant research efforts have been dedicated to developing

Received July 31, 2018; revised Jan. 28, 2019; accepted Jan. 29, 2019

Author contributions: C.L. wrote the first draft of the paper; C.L., B.N., R.G.-S., and M.A.H. edited the paper; C.L., R.G.-S., and M.A.H. designed research; C.L., M.A.R., X.Y.H., B.N., and R.G.-S. performed research; C.L., B.N., R.G.-S., and M.A.H. analyzed data; C.L., R.G.-S., and M.A.H. wrote the paper.

This work was supported by a University of Queensland Research Scholarship to C.L. and X.Y.H.; a University of Queensland Development Fellowship to M.A.R.; a Human Frontier Science Program Fellowship LT000762/2012 to R.G.S.; the National Health and Medical Research Council (Project Grants 1068871 and 1129367; Senior Research Fellowship 1111042 to M.A.H.; and Project Grants 1101974 and 1099690 to B.N.); and the Australian Research Council (ARC Discovery Project Grant 160104359). The LSM 710 META confocal microscope used in this study was supported by ARC Grant LIEF LE130100078. Some strains of C. elegans were provided by the Caenorhabditis Genetic Center (CGC), which is funded by National Institutes of Health's Office of Research Infrastructure Programs (Grant P40 0D010440). We thank Luke Hammond and Rumelo Amor for help with microscopy, Rowan Tweedale and members new methods to repair an axon following transection because current outcomes are less than optimal. A novel approach to this problem has come from work in invertebrate systems, in which some neurons are capable of spontaneously rejoining the two separated axonal fragments after transection. This regenerative mechanism, called axonal fusion, has been observed in a number of invertebrate species (Hoy et al., 1967; Birse and Bittner, 1976; Deriemer et al., 1983; Macagno et al., 1985; Bedi and Glanzman,

of the Hilliard laboratory for helpful discussion and comments; Guangshuo Ou for the p0G172 plasmid; Nick Valmas for the PNV MYR::mCherry plasmid; and Elia Di Schiavi for advice regarding cell-specific RNAi.

Correspondence should be addressed to Massimo A. Hilliard at m.hilliard@uq.edu.au or Rosina Giordano-Santini at r.giordanosantini@uq.edu.au.

https://doi.org/10.1523/JNEUROSCI.1952-18.2019

Copyright $\odot 2019$ the authors 
2001), but has been best characterized in the mechanosensory neurons of the nematode Caenorhabditis elegans (Ghosh-Roy et al., 2010; Neumann et al., 2011, 2015; Abay et al., 2017; Basu et al., 2017). To undergo axonal fusion, the proximal axon (still attached to the cell body) must first regrow and then get in close proximity to its separated axonal fragment (reconnection). Once reconnection has occurred, the two axonal fragments can fuse their membranes to reestablish membrane and cytoplasmic continuity (axonal fusion). We and others have shown in C. elegans that this fusion not only restores continuity of the axon following UV laser axotomy (Neumann et al., 2011), but also restores neuronal function (Abay et al., 2017; Basu et al., 2017).

The key molecular effector of axonal fusion is the C. elegans membrane fusogen epithelial fusion failure-1 (EFF-1). EFF-1, a nematode-specific transmembrane glycoprotein with structural and functional similarity to class II viral fusion proteins (PérezVargas et al., 2014), functions to merge closely apposed plasma membranes. EFF-1 activity in the injured neuron is crucial for successful axonal fusion; eff-1 mutant animals exhibit severe axonal fusion defects (Ghosh-Roy et al., 2010; Neumann et al., 2015), which can be rescued by expression of WT EFF-1 selectively in the injured neuron, revealing its cell-autonomous function (Neumann et al., 2015). There is strong evidence that the activity of EFF-1 is controlled via its dynamic subcellular localization. To mediate fusion, EFF-1 must be inserted into the membrane and it is inactive when sequestered in intracellular compartments. This was first demonstrated in other cell types in C. elegans, including the hypodermis, where EFF-1 mediates cellcell fusion for the formation of syncytia during development (Mohler et al., 2002). In these cells, EFF-1 is mobilized from intracellular compartments to the plasma membrane, where it mediates fusion (del Campo et al., 2005; Smurova and Podbilewicz, 2016). Similarly, in the posterior lateral microtubule (PLM) mechanosensory neurons, EFF-1 exists largely within puncta in the steady state, but following injury is mobilized to the regenerating axonal membrane (Neumann et al., 2015). How these changes in EFF-1 localization and activity are regulated within the neuron is currently unknown, but they likely represent a critical step in the control of axonal fusion.

To date, the only other molecules implicated in C. elegans axonal fusion are components of the apoptotic clearance machinery (Neumann et al., 2015) and the conserved microRNA let-7, which functions to regulate this pathway (Basu et al., 2017). The clearance signaling pathways were first described as a mechanism for apoptotic cell corpse engulfment (Liu and Hengartner, 1998; Wu and Horvitz, 1998; Wang et al., 2003, 2010; Zhang et al., 2012), but appear to have been repurposed for axonal fusion, and function upstream of EFF-1 to promote recognition of the severed axonal fragment. Axonal injury triggers exposure of the phospholipid phosphatidylserine (PS) on the surface of the severed axon. PS acts as a "save me" signal by recruiting both the PS receptor PSR-1, which is present on the growth cone of the regrowing fragment, and the secreted PS-binding protein TTR-52, which initiates signaling to improve the efficiency of axonal fusion (Neumann et al., 2015; Abay et al., 2017). Animals with mutated $p s r-1$ or ttr-52 exhibit axonal fusion defects, which can be rescued with overexpression of EFF-1, indicating that EFF-1 acts genetically downstream of these recognition molecules (Neumann et al., 2015).

However, axonal fusion must also involve additional, undiscovered molecules. In the absence of the apoptotic genes, axonal fusion can still occur at a low level and EFF-1 is still mobilized to the membrane, indicating the existence of mechanisms that can bypass the apoptotic recognition machinery to enable fusion. Here, we identify a novel regulator of axonal fusion. We reveal that the GTPase RAB-5 can negatively regulate axonal fusion by functioning within the injured neuron and present evidence that this protein controls the level of EFF-1 on the neuronal membrane via endocytosis. Therefore, we propose a model in which recycling of EFF-1 via RAB-5 is a critical mechanism for the control of fusogen function and can regulate axonal fusion as a mechanism of repair.

\section{Materials and Methods}

Strains and genetics. Standard techniques were used for C. elegans strain maintenance and genetic manipulations (Brenner, 1974). All experiments were performed at $22^{\circ} \mathrm{C}$ (room temperature) on $\mathrm{L} 4$ hermaphrodites animals unless otherwise specified. The following mutations were used: rab-10(dx2) I, eff-1(ok1021) II, ehs-1(ok146) II, psr-1(ok714) IV, dyn-1(ky51)X. The integrated transgenic strain QH3135 [zdIs5(Pmec-4:: GFP) I] was used as a background strain for performing axotomies. The transgenic strain QH4748 [eff-1(ok1021) II; vdEx662[Pmec-4::eff-1::gfp; Pmec-4::mCherry; Podr-1::DsRed]] (Neumann et al., 2015) was used as a background strain for all experiments involving EFF-1 confocal imaging of uncut and axotomized animals. To generate extrachromosomal arrays, microinjections were performed into the germline using standard methods (Mello and Fire, 1995). The following transgenes were generated (concentrations used for the microinjection mix are indicated in brackets; all injection mixes had a total concentration made up to $100 \mathrm{ng} / \mu \mathrm{l}$ using empty pSM plasmid): $v d E x 1192 / v d E \times 1193 /$

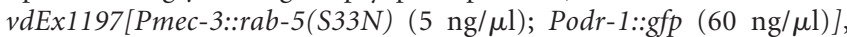
$v d E x 1450 / v d E x 1451 / v d E x 1452$ [Pmec-3::rab-5(Q78L) (5 ng/ $\mu$ l); Podr-

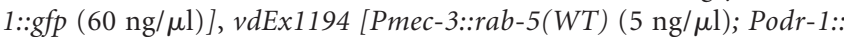

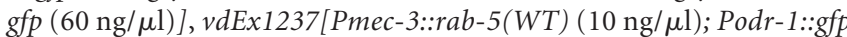
(60 ng/ $\mu \mathrm{l})], v d E x 1051 / v d E x 1055 / v d E x 1084[$ Pmec-3s::rab-5(sense) (5

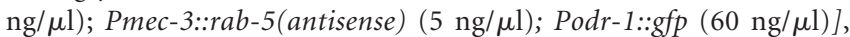
$v d E x 1301 / v d E x 1302 / v d E x 1303$ [Pmec-3::rab-7(T23N) (5 ng/ $\mu$ l); Podr$1:: g f p \quad(60 \mathrm{ng} / \mu \mathrm{l})], \quad v d E x 1375 / v d E x 1390 / v d E x 1443[P m e c-3:: r a b-$

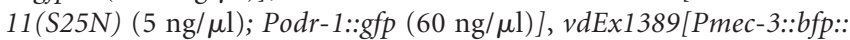

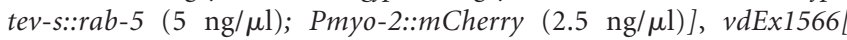

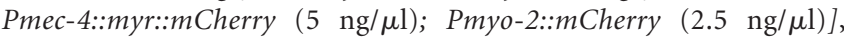
vdEx1576 [Pmec-4::myr::mCherry (15 ng/ $\mu \mathrm{l})$; Pmyo-2::mCherry $(2.5$ $\mathrm{ng} / \mu \mathrm{l})]$. It should be noted that a TEV-S signal exists between the BFP and RAB-5 sequences in $v d E x 1389$; this signal allows for protein cleavage with addition of TEV protease and is therefore highly unlikely to have affected the outcome of the current study.

Molecular biology. Standard molecular biology techniques were used (Sambrook et al., 1989). To generate the plasmid Pmec-3::rab-5(WT), the rab-5 gene was amplified from the pCL206 plasmid using $5^{\prime}$ gcTCTAGAatggccgcccgaaacgcagg- $3^{\prime}$ and $5^{\prime}$-gggaCCCGGGttatttacagcat gaaccc- $3^{\prime}$. These primers introduced XbaI and XmaI restriction sites, respectively, which were used to clone the amplicon into the plasmid L1026 (pPD57_56 was a gift from Andrew Fire (Addgene plasmid \# 1452; http://n2t.net/addgene:1452; RRID:Addgene_1452)). The QuikChange II Site-Directed Mutagenesis Kit (Agilent Technologies) was then used to generate rab-5(Q78L) (CA) and rab-5(S33N) (DN) variants of this plasmid, using the primers $5^{\prime}$-aaatctgggatactgcaggaaaagaaagatatcattattgg- $3^{\prime}$, $5^{\prime}$-ccaatgaatgatatctttcttttcctgcagtatcccgattt- $3^{\prime}$ and $5^{\prime}$-ctatcatttcaggcaaaa actctctcgtattgcgattc- $3^{\prime}, 5^{\prime}$-gaatcgcaatacgagagagtttttgcctgaaatgatag- $3^{\prime}$ respectively.

A PCR fusion approach was used to generate cell-specific rab-5 sense and antisense molecules Pmec-3s::rab-5(sas) (Esposito et al., 2007). rab-5 was amplified from genomic C. elegans DNA using standard primers (5'-cgtgccttcaatctttttcg- $3^{\prime}$ and $5^{\prime}$-acaatgacgacgatcacaggc- $\left.3^{\prime}\right)$. Pmec-3s was amplified from the L3784 plasmid (Pmec-3::gfp, L3784 was a gift from Andrew Fire (Addgene plasmid \# 1591; http://n2t.net/addgene:1591; RRID: Addgene_1591)) with a standard forward primer (5' -aggtacccggagtagtt ggc- $\left.3^{\prime}\right)$ and two different reverse primers with sequences complementary to the extremities of rab-5 at the $5^{\prime}$ ends ( $5^{\prime}$-atgttgcatttttctttcca gaatctataacttgatagcgata- $3^{\prime}$ and $5^{\prime}$-cttcccaactaccatgtacaaaatctataacttgatagc gata- $\left.3^{\prime}\right)$. The rab-5 and Pmec-3s reaction products were then fused using 
nested primers $5^{\prime}$-ggcagtaatgaagacgtccat- $3^{\prime}$ and $5^{\prime}$-gaagggttgatg gtacatgaaa- $3^{\prime}$ or $5^{\prime}$-ttctggaaagaaaaatgcaacat- $3^{\prime}$.

Pmec-3::rab-7(T23N) (DN) and Pmec-3::rab-11(S25N) (DN) were generated using the QuikChange II Site-Directed Mutagenesis Kit on Pmec-3::rab-7 and Pmec-3::rab-11 plasmids, with primers 5'-cgggcg ttggaaagaattctttgatgaatcaatatg- $3^{\prime}, \quad 5^{\prime}$-catattgattcatcaaagaattctttccaacg cccg- $3^{\prime}$ and $5^{\prime}$-gagactcaggcgtcggaaagaataatctcctgtctcgtttcac- $3^{\prime}, 5^{\prime}$-gtgaaa cgagacaggagattattctttccgacgcctgagtctc- $3^{\prime}$, respectively. The construction of Pmec-3::rab-7 and Pmec-3::rab-11 involved amplification of each gene from C. elegans genomic DNA with primers to introduce XbaI and XmaI restriction sites, followed by cloning into the L1026 plasmid downstream of Pmec-3.

To generate Pmec-3::bfp::tev-s::rab-5, the bfp::tev-s::rab-5 insert was amplified from pOG172, a kind gift from Prof. Guangshuo Ou (Tsinghua University, Beijing). Primers were used to introduce BamHI and MscI restriction sites for cloning into L1026 downstream of Pmec-3. To generate the Pmec-4::myr::mCherry plasmid, myr::mCherry from the PNV:: myr::mCherry plasmid was cloned into Pmec-4::GFP using MscI and EcoRI restriction enzymes that remove the GFP sequence.

Laser axotomy. We performed UV laser axotomy of PLM in animals at the L4 larval stage as described previously (Neumann et al., 2011, 2015). Animals were anesthetized using $0.05 \%$ tetramisole hydrochloride on $3 \%$ agarose pads. The axotomy was performed $\sim 50 \mu \mathrm{m}$ from the cell body using a MicroPoint Laser System Basic Unit attached to a Zeiss Axio Imager A1. At $48 \mathrm{~h}$ after axotomy, animals were analyzed on a Zeiss Axio Imager Z1 equipped with a Photometrics Cool Snap HQ2 camera with MetaMorph software for the presence of reconnection and fusion. If it was unclear whether a distal fragment had been maintained, then the animal was scored again at $72 \mathrm{~h}$ after axotomy.

Confocal microscopy. Localization studies of EFF-1 and RAB-5 were performed on a LSM 710 META confocal microscope equipped with a GaAsP detector and Zen 2012 software. L4 animals were mounted on 3\% agarose pads in $25 \mathrm{~mm}$ sodium azide. Separate $Z$-stacks were performed of the PLM cell body and proximal axon. For imaging of EFF-1::GFP and cytoplasmic mCherry, green fluorescence was visualized with a $488 \mathrm{~nm}$ laser (5\% power for the axon, $2 \%$ power for the cell body; gain of 600 and $4 \times$ averaging for both) and red fluorescence was visualized with a 543 $\mathrm{nm}$ laser ( $1 \%$ power for the axon, $0.2 \%$ power for the cell body; gain of 500 and $4 \times$ averaging for both). For imaging of BFP::TEV-S::RAB-5, blue fluorescence was visualized with a $405 \mathrm{~nm}$ laser $(0.5 \%$ power, gain of $500,4 \times$ averaging). To image MYR::mCherry, red fluorescence was again visualized with a $543 \mathrm{~nm}$ laser (up to $10 \%$ power, gain of $500,4 \times$ averaging).

To image EFF-1::GFP and BFP::RAB-5 after axotomy, animals were mounted for axotomies in tetramisole as described above. They were then recovered in drops of M9 buffer onto seeded NGM plates for either 3 or $6 \mathrm{~h}$, after which they were mounted in sodium azide for confocal imaging.

Vesicle time-lapse imaging. For characterization of EFF-1::GFP vesicle dynamics, animals were mounted on $0.05 \%$ tetramisole. $Z$-stacks of the PLM cell body were acquired at $30 \mathrm{~s}$ intervals for a total of $5 \mathrm{~min}$ using a spinning-disk confocal system (Marianas; Intelligent Imaging Innovations) consisting of an Axio Observer Z1 (Carl Zeiss) equipped with a CSU-W1 spinning disk head (Yokogawa Electric), sCMOS camera (ORCA-Flash4.0 V2 Hamamatsu Photonics), and a 100×/1.46 numerical aperture oil-immersion objective. Image acquisition was performed using SlideBook version 6.0 (Intelligent Imaging Innovations) and sampling distances of $x-y=63 \mathrm{~nm}$ and $z=130 \mathrm{~nm}$. Green fluorescence was visualized with a $488 \mathrm{~nm}$ laser (100 ms exposure) and red fluorescence was visualized with a $543 \mathrm{~nm}$ laser (50 ms exposure). Images were deconvolved with Huygens Professional version 18.04 (Scientific Volume Imaging) run on a GPU-accelerated computer $(3 \times$ NVIDIA Tesla V100) using the CMLE algorithm, with a signal-to-noise ratio of 20, a background of 100, and 40 iterations. Vesicles were classified as immobile or mobile based on the presence of any movement in this timeframe. Mobile vesicles ( 29 of 42) were measured for movement in the $x-y$ axis per frame by drawing a linear region of interest from the center of the vesicle to the center at its subsequent location; the total distance was averaged over the time imaged to be expressed in micrometers per minute. dyn-1 temperature shifts. To test the temperature-sensitive allele $d y n$ 1(ky51), temperature shifts were performed for either $30 \mathrm{~min}$ or $2 \mathrm{~h}$. L4 animals were placed on NGM plates in a $25^{\circ} \mathrm{C}$ incubator. Mutant and control animals were shifted to $25^{\circ} \mathrm{C}$ concurrently on separate plates and mounted on the same slide for subsequent confocal imaging. $d y n$ $1(k y 51)$ animals raised at $25^{\circ} \mathrm{C}$ did not lay viable eggs, whereas those laid at $15^{\circ} \mathrm{C}$ and then transferred to $25^{\circ} \mathrm{C}$ developed to the $\mathrm{L} 4$ stage. However, the animals raised at $25^{\circ} \mathrm{C}$ demonstrated nonspecific increases in EFF-1: GFP intensity in both mutant and control groups (data not shown). Temperature shifts used in this assay were therefore limited to a maximum of $2 \mathrm{~h}$.

Confocal image analysis. Image analysis was performed using Fiji for Mac OS X (ImageJ). To score for the presence of EFF-1::GFP on the membrane of the cell body, fluorescence profiles of line scans were obtained using the "Plot Profile" tool in ImageJ. EFF-1::GFP was scored as localizing to the membrane if the peak of green intensity (EFF-1::GFP) and the peak of red intensity (cytoplasmic mCherry) did not overlap.

For EFF-1::GFP intensity calculations, average projections of $Z$-stacks were analyzed. For the axon, a line scan was performed along the axon for the initial $\sim 50 \mu \mathrm{m}$ anterior to the cell body and the mean intensity in the green and red channels was measured. Background fluorescence was calculated using the same line scan moved to three different positions around the axon in the image; the mean background fluorescence from these three readings was subtracted from the average intensity value of the axon. The ratio of the GFP intensity to the mCherry intensity was then calculated to control for differences in transgene expression between animals. For intensity calculations of the cell body, an ROI was drawn following the boundary of the cell body and calculations were performed as for the axon.

For measurements of EFF-1::GFP border localization, average projections of $Z$-stacks were analyzed. An ROI was drawn along the border of the PLM cell body. The average GFP intensity was first measured along this line (border intensity), after which the average GFP intensity was measured within the full area (cell body intensity). The final measurement was expressed as a ratio of the average border intensity to cell body intensity. Background subtraction was performed as described for intensity measurements.

To quantify EFF-1::GFP and BFP::RAB-5 puncta size and number, maximum projection confocal images of $Z$-stacks were analyzed. Puncta were identified automatically using the ImageJ plugin Squassh (Rizk et al., 2014). The following settings were used: background removal, rolling ball window size 10 , regularization 0.1 , minimum object intensity 0.3 , subpixel segmentation, automatic local intensity estimation, Poisson noise model, and Gaussian psf approximation as for confocal microscopy. The circularity of the resulting objects was calculated using the "Analyze Particles" function in ImageJ. The following exclusion criteria were applied to all objects: circularity $<0.7$ and object located in the proximal axon or outside the neuron. If none of the puncta selected in a cell body met these criteria, then the cell body was discarded from the analysis.

For colocalization studies of EFF-1 and RAB-5, only enlarged puncta were analyzed; these were defined as puncta $>125$ units because puncta of this size were specific to RAB- 5 overactivity and were never observed in nontransgenic controls. Squassh analysis was applied to both the green and blue channels, providing colocalization values for the selected objects. Identical settings (described above) were used for the two channels.

Statistical testing. Statistical analysis was performed using GraphPad Prism and Microsoft Excel. Axotomy data were analyzed using the Fischer's exact test. All other data were analyzed using either the $t$ test or the $t$ test with Welch correction (the latter was performed if the SDs of two compared groups were significantly different). For all experiments performed using transgenic animals, nontransgenic siblings from the same isolate were used as a control group. For experiments testing the effect of a mutant allele, WT animals were used as a control group.

\section{Results}

RAB-5 regulates the efficiency of regenerative axonal fusion

To identify molecules with a role in axonal fusion, we used a candidate gene approach to find suppressors of the fusion defect of psr-1 mutant animals. We considered a suppressor to be any 
a
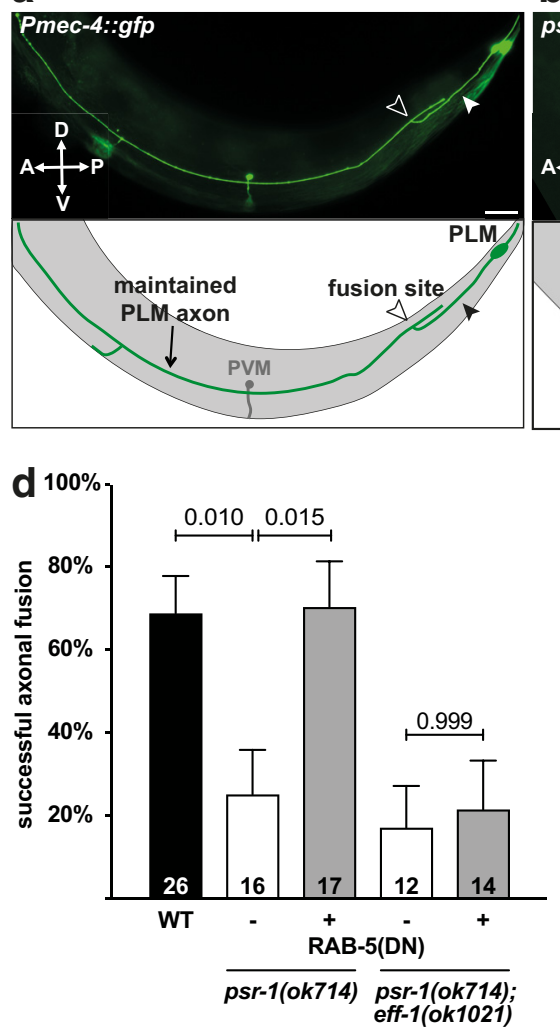

b

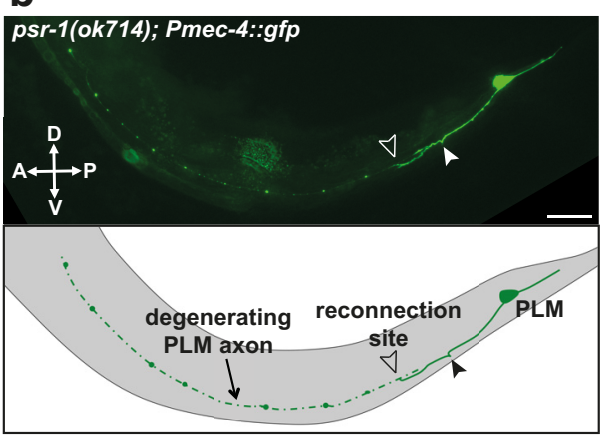

e

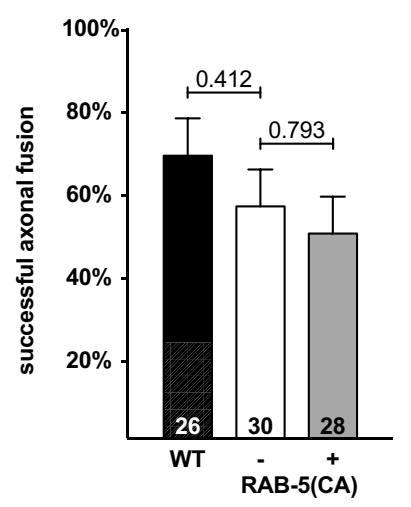

c

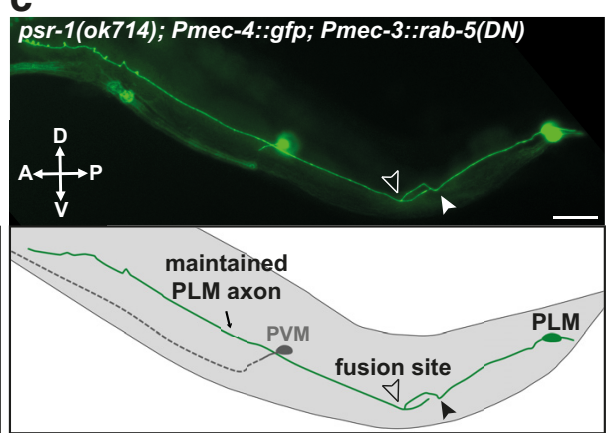

f
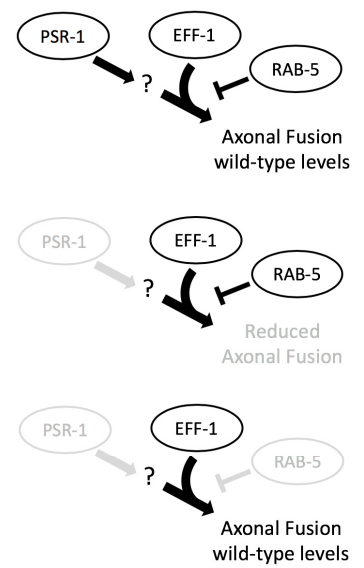

Figure 1. Perturbing RAB-5 activity increases EFF-1-dependent axonal fusion. $\boldsymbol{a}$, Successful PLM axonal fusion in a WT animal (Pmec-4::GFP) $48 \mathrm{~h}$ after axotomy. The filled arrowhead indicates the cut site; the open arrowhead indicates the site of fusion. Another neuron (PVM, gray in schematic) is visible in the midbody of the animal. $\boldsymbol{b}$, Defective PLM axonal fusion in a $p s r-1$ (ok714) animal $48 \mathrm{~h}$ after axotomy. The filled arrowhead indicates the cut site; the open arrowhead indicates the site of reconnection (which did not result in fusion). $c$, Rescue of PLM axonal fusion in a psr-1(ok714) animal by expression of RAB-5(DN) $48 \mathrm{~h}$ after axotomy. The filled arrowhead indicates the cut site; the open arrowhead indicates the site of fusion. PVM (gray in schematic) can be seen but is out of focus. $\boldsymbol{d}$, Quantification of axonal fusion in psr-1 (ok714) animals expressing RAB-5(DN) in the PLM neuron, demonstrating rescue of the psr-1(ok714) axonal fusion defect. This rescue is suppressed in psr-1 (ok714); eff-1(ok1021) mutant animals, indicating that the effect of RAB-5(DN) is eff-1 dependent. $\boldsymbol{e}$, Quantification of axonal fusion in animals expressing RAB-5(CA) in the PLM neuron shows no significant effect on the level of axonal fusion compared with controls. Note that the same WT dataset is used for both $\boldsymbol{d}$ and $\boldsymbol{e}$. Error bars indicate the SE of proportions; $n$ values are listed below each bar; exact $p$-values from Fischer's exact test. D, Dorsal; $V$, ventral; $A$, anterior; P, posterior for all images. Scale bars, $25 \mu$ m. $\boldsymbol{f}$, Schematic illustrating how PSR-1 and RAB- 5 act genetically upstream of EFF-1 in axonal fusion, with PSR-1 promoting fusion and RAB-5 suppressing EFF-1 availability on the membrane. Loss of function in psr- 1 thus leads to reduced fusion efficiency, whereas concurrent perturbation of RAB- 5 is able to restore fusion to normal levels.

gene in which a loss-of-function, gain-of function, or overexpression could overcome the $p s r-1$ mutant defect and improve axonal fusion. This allowed us to assess whether a candidate gene could effectively bypass the apoptotic recognition machinery and modulate the efficiency of axonal fusion.

Our primary candidate was the endocytic GTPase RAB- 5 because this protein has been shown to modulate the efficiency of fusion in other C. elegans tissues. Specifically, loss-of-function or depletion of RAB-5 leads to a hyperfusion phenotype in the $C$. elegans hypodermis (Smurova and Podbilewicz, 2016). We first investigated the effect of perturbing RAB-5 activity by performing axotomies on the mechanosensory neurons of psr-1 mutant animals expressing a dominant-negative version of RAB-5 [RAB$5(\mathrm{DN})]$. Axotomies were performed on the PLM neurons $\sim 50$ $\mu \mathrm{m}$ anterior to the cell body, as described previously (Neumann et al., 2011, 2015). The efficiency of axonal fusion was calculated from axons that regrew and visibly reconnected to their distal fragment. Successful axonal fusion occurred if the distal fragment was maintained $48 \mathrm{~h}$ after axotomy (Fig. $1 \mathrm{a}$ ); fusion was considered unsuccessful if the distal fragment instead underwent degeneration (Fig. 1b).

As shown previously (Neumann et al., 2015), psr-1 mutant animals displayed a defect in axonal fusion, with a much lower efficiency compared with WT animals. Remarkably, we found that expression of RAB-5(DN) in the PLM neuron was sufficient to rescue this defect to WT levels (Fig. $1 c, d, f$ ). We also performed axotomies on WT animals expressing constitutively active RAB-5 [RAB-5(CA)] in the mechanosensory neurons, hypothesizing that this increased RAB-5 activity might generate the opposite phenotype and reduce the efficiency of axonal fusion. However, although there was a trend toward reduction, there was no significant change in axonal fusion efficiency in these animals (Fig. 1e).

Given that EFF-1 is the key molecular effector of this process, we next investigated whether RAB-5 modulates axonal fusion through an interaction with EFF-1. To address this, we performed axotomies on double mutant animals of eff-1- and psr-1expressing RAB-5(DN). We found that loss of eff- 1 suppressed the increase in axonal fusion mediated by RAB-5(DN) (Fig. 1d), indicating that RAB-5(DN) increases the axonal fusion efficiency in an eff-1-dependent manner (Fig. $1 f$ ).

RAB-5 controls EFF-1 localization to the plasma membrane We next investigated how RAB-5 was regulating the activity of EFF-1 in mediating axonal fusion. We suspected that perturbing RAB- 5 prevented the endocytosis of EFF-1, which would increase 
the amount of EFF-1 available at the membrane and enhance its activity. RAB-5 and its mammalian ortholog Rab5 are known to localize to early endosomes and play important roles in endocytosis. They facilitate the transport of clathrin-coated vesicles to early endosomes, fusion between endosomes, and cargo trafficking from endosomes into lysosomes for degradation (Gorvel et al., 1991; Bucci et al., 1992; Zerial and McBride, 2001; Hutagalung and Novick, 2011; Zeigerer et al., 2012). Correspondingly, altering RAB-5 activity produces specific cellular phenotypes: perturbing RAB-5 activity using RAB-5(DN) inhibits endocytosis and causes membranous accumulation of proteins (Stenmark et al., 1994), whereas expression of RAB-5(CA) leads to excessive early endosome fusion and the presence of enlarged early endosomes (Stenmark et al., 1994; Patton et al., 2005). In the $C$. elegans hypodermis, depletion or loss-of-function of RAB-5 results in mislocalization of EFF-1 to the plasma membrane, which in turn is associated with excessive hypodermal cell-cell fusion (Smurova and Podbilewicz, 2016). We therefore predicted that perturbing RAB-5 activity in the PLM neurons would result in similar changes to EFF-1 localization.

To visualize EFF-1 within the PLM neuron, we used a transgenic strain in which eff-1-null mutant animals express cytoplasmic mCherry in the PLM mechanosensory neurons, as well as GFP-tagged EFF-1. We previously demonstrated that this EFF$1::$ GFP transgene is functional and sufficient to rescue axonal fusion defects in eff-1 mutant animals (Neumann et al., 2015). Using confocal microscopy, we characterized EFF-1 localization in the cell body and proximal axon of PLM (Fig. 2a-d). In the uninjured, WT axon, EFF-1 formed an indiscriminate punctate pattern as we previously reported (Neumann et al., 2015) (Fig. $2 b)$. In the cell body, it was also present as intracellular puncta, with no clear localization to the cell membrane (Fig. $2 c, d$ ). We chose to perform subsequent localization studies specifically in the cell body because this wider structure allows for any mobilization of EFF-1 to the membrane to be clearly identified.

To determine whether perturbing RAB-5 activity led to EFF-1 localization at the membrane, we coexpressed RAB-5(DN). In these animals, EFF-1::GFP formed a more continuous pattern along the axon (Fig. $2 b$ ) and accumulated at the membrane of the PLM cell body (Fig. $2 c, d$ ), a process that was commonly associated with membranous protrusions of EFF-1 (Fig. $2 c$, arrowhead). Line scan profiles through these protrusions clearly demonstrated that EFF-1 was present on the membrane of the cell body (with a fluorescence peak occurring well outside the margin of the cytoplasmic mCherry signal), a phenomenon that we never observed in control animals (Fig. $2 c, d$ ). To quantify EFF-1 localization, we measured the average intensity of GFP in the membrane of the cell body normalized to the average intensity for the whole cell body (see Materials and Methods section). This revealed a significant increase in the relative amount of EFF-1::GFP at the membrane in the presence of RAB-5(DN) (Fig. 2e). These results indicate that neuronal-specific loss of RAB- 5 activity leads to EFF-1 accumulation at the membrane of the neuronal cell body.

Enhancing RAB-5 activity, through either overexpression of RAB-5(WT) or expression of RAB-5(CA) had no effect on the relative amount of EFF-1::GFP at the cell membrane (Fig. 2e). This was consistent with our axotomy results, which indicated that increased RAB-5 activity did not significantly alter EFF-1 function. Together, these findings suggest that RAB-5 activity functions directly or indirectly in the endocytosis of EFF-1 molecules that have been mobilized to the membrane.

We showed previously that axotomy of the PLM neuron leads to the rapid accumulation of EFF-1 on the proximal and distal stumps, and on the growth cone of the regenerating axon (Neumann et al., 2015). Here, we tested whether EFF-1 localization also changed in the PLM cell body following axotomy and found only a minor shift to the cell periphery at $6 \mathrm{~h}$ after surgery (Fig. $2 c-e$ ).

\section{Plasma membrane accumulation of EFF-1 forms extracellular vesicles}

Interestingly, we observed that animals expressing RAB-5(DN) not only had protrusions of the transmembrane EFF-1::GFP from the PLM cell body, but also generated what appeared to be extracellular EFF-1::GFP-positive vesicles (Fig. 3, Movies 1, 2). These extracellular vesicles, ranging in number from 2 to 20 per neuron, were present around the PLM cell body and proximal axon and were reproducible in multiple independent transgenic strains (Fig. 3a). These vesicles appeared to be exclusive to the expression of RAB-5(DN) because we did not observe any vesicles in eff-1 mutant animals expressing EFF-1::GFP in PLM (EFF-1 rescue) (Fig. 3a), nor in WT animals carrying the same transgene (EFF-1 overexpression) (data not shown). Intriguingly, previous work has shown the presence of EFF-1 vesicles in cultured medium of baby hamster kidney cells transfected with EFF-1 (Zeev-Ben-Mordehai et al., 2014), and vesicles containing AFF-1, a second C. elegans fusogen, have been described both in vitro from mammalian cells (Avinoam et al., 2011) and in vivo from seam cells (Oren-Suissa et al., 2017) (see Discussion).

To determine the composition of these vesicles, we generated transgenic strains that coexpress a cytoplasmic marker (mCherry) and a membrane-bound marker (MYR::mCherry) in addition to EFF-1::GFP and RAB-5(DN). Vesicles observed in these animals presented no detectable mCherry signal (Fig. $3 b$ ), indicating that membrane-bound forms of mCherry were excluded from the vesicles and that they contained a highly reduced volume of cytoplasm (based on the resolution of confocal imaging). This suggests that these vesicles may be selective in their composition and contain mostly EFF-1, similar to those vesicles reported in cell culture (Zeev-Ben-Mordehai et al., 2014).

To characterize the dynamics of the vesicles, we undertook time-lapse imaging of the PLM cell body. Confocal imaging of the vesicles at $30 \mathrm{~s}$ intervals for up to 5 min revealed that they fell into two categories of mobility: "mobile" vesicles, representing approximately two-thirds of the vesicles, which exhibited linear or disorganized, oscillatory movements around the cell body (Movies 1,2), and "immobile" vesicles, which exhibited no movement in this time period (Fig. $3 d$ ). Mobile vesicles exhibited movement at an average rate of $1.08 \pm 0.2 \mu \mathrm{m} / \mathrm{min}(n=29)$. We observed that these vesicles are present from the first and second larval stages. Budding of the vesicles from the cell body was observed on several occasions at the fourth larval stage (Fig. $3 d$ ). EFF-1 retained at the membrane also underwent dynamic changes and the growth and movement of large protrusions of EFF-1 from the cell body was captured within a period of minutes (Fig. 3c, Movie 1).

To determine whether neuronal injury can affect the number of vesicles either in the presence or absence of RAB-5(DN), we performed axotomies and visualized the axon and cell body at 3 and $6 \mathrm{~h}$ after axotomy in these two different backgrounds. In WT animals, we only observed one out of 29 animals with vesicles in proximity of the PLM cell body (data not shown). In RAB-5(DN) animals, we found that axotomy did not significantly change the number of vesicles secreted compared with unoperated animals (average of $8.5 \pm 1.4,6.6 \pm 0.9$, and $5.5 \pm 1.4$ vesicles per cell 
a
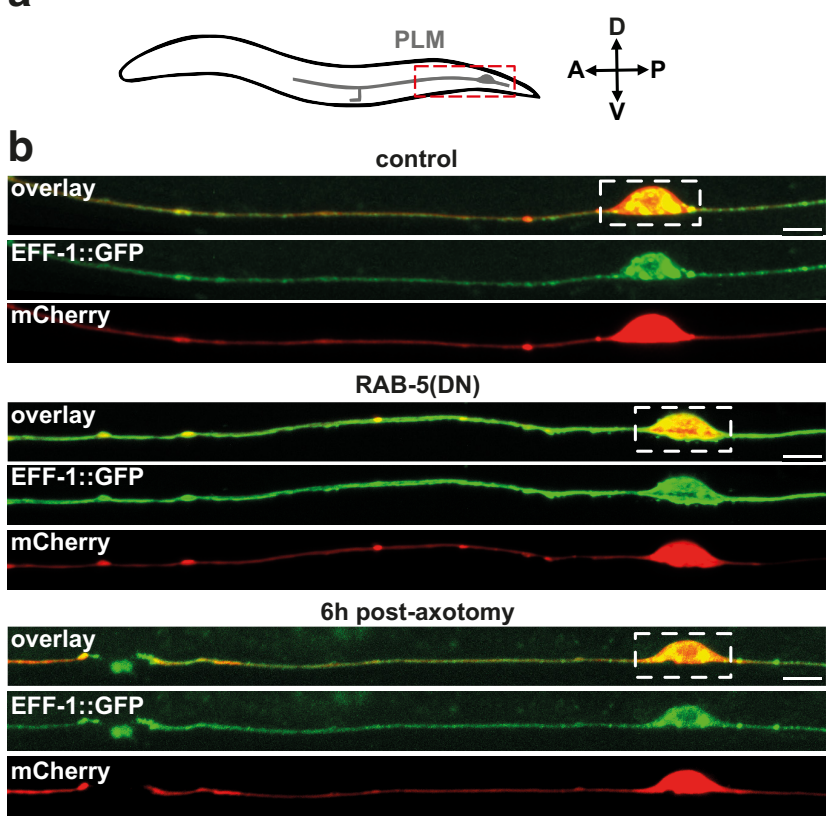

C
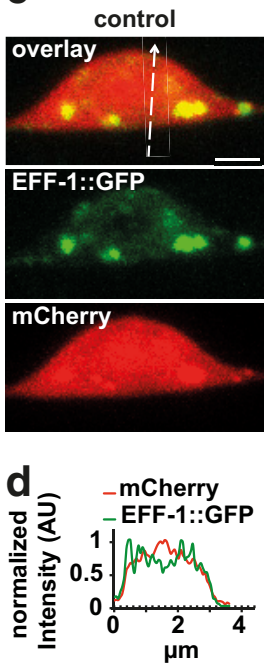

RAB-5(DN)
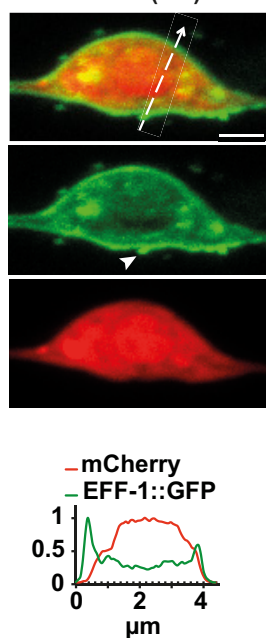

$6 \mathrm{~h}$ post-axotomy
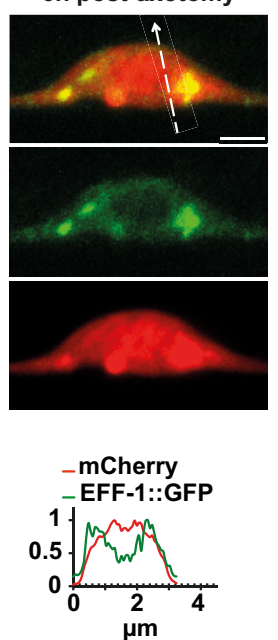
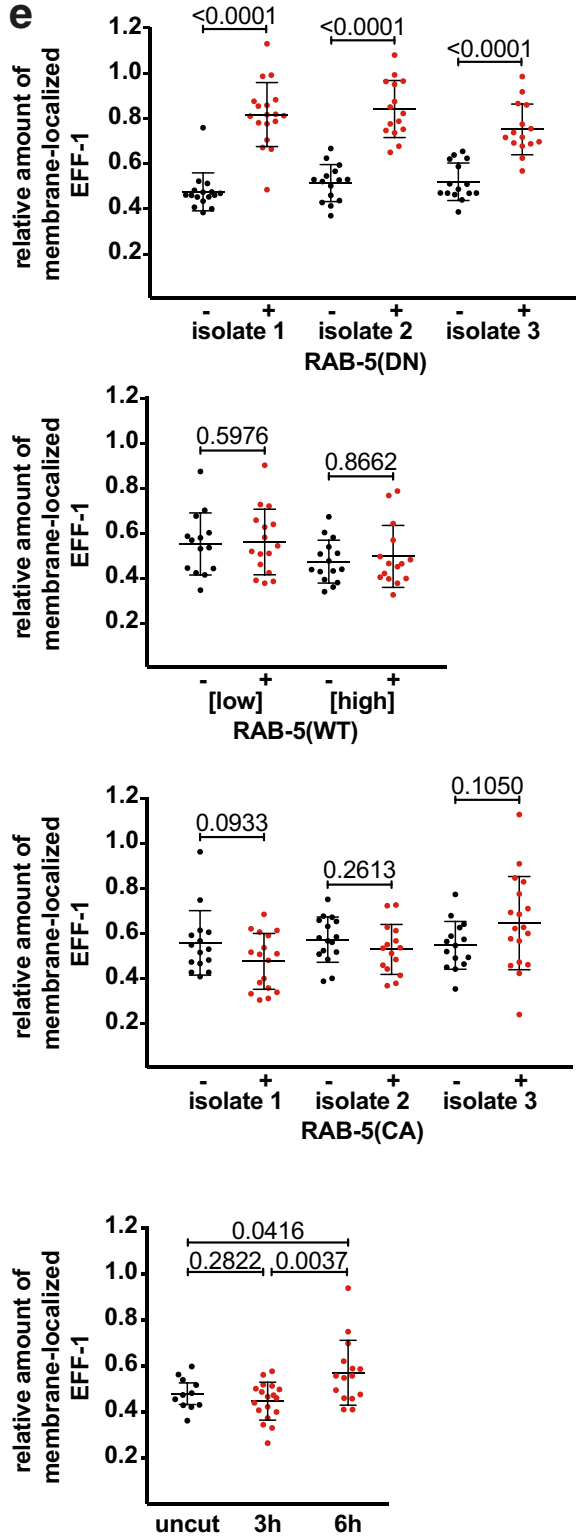

Figure 2. RAB-5 controls EFF-1 localization to the plasma membrane. $\boldsymbol{a}$, Schematic of the PLM neuron in the tail of the animal; dashed box indicates the region depicted in $\boldsymbol{b}$. $\boldsymbol{b}$, Representative maximum projection confocal images of the PLM neuron in eff-1(ok1021) animals expressing EFF-1::GFP and cytoplasmic mCherry in PLM. A control animal (top) is compared with an animal expressing RAB-5(DN) (middle) and an animal at $6 \mathrm{~h}$ after axotomy (bottom). Dashed boxes indicate the regions magnified in $\mathbf{c}$. Scale bars, $5 \mu$ m. $c$, Magnification of the PLM cell bodies in $\boldsymbol{b}$. In the animal expressing RAB-5(DN) (middle), EFF-1::GFP localizes to a membranous protrusion (arrowhead). Dashed line arrows indicate the position of a line scan drawn through each cell body, with the fluorescence profile from each line depicted in $\boldsymbol{d}$. Contrast settings have been adjusted to better visualize EFF-1 in the cell body rather than the axon. Scale bars, $1 \mu \mathrm{m}$. $\boldsymbol{d}$, Fluorescence profiles from line scans (dashed line arrows) of the overlying cell bodies [control animal on left; RAB-5(DN) animal in middle, animal $6 \mathrm{~h}$ after axotomy on right]. The presence of an EFF-1::GFP fluorescence peak outside of the margin of the cytoplasmic mCherry signal confirms membranous EFF-1::GFP localization in the presence of RAB-5(DN), but not in the control animals or animals after axotomy. Results are demonstrative of $\geq 10$ animals per group. AU, Arbitrary units. $\boldsymbol{e}$, Quantification of the relative amount of EFF-1::GFP at the membrane of the cell body in eff-1(ok1021) animals coexpressing EFF-1::GFP in three different RAB-5 backgrounds, and wild-type axotomized animals. Top row: RAB-5(DN), second row: RAB-5(WT) (low $(5 \mathrm{ng} / \mu \mathrm{l})$ and high (10 $\mathrm{ng} / \mu \mathrm{l})$ injection concentrations are shown), third row: RAB-5(CA), and bottom row: animals at 3 and $6 \mathrm{~h}$ after axotomy. For each independent transgenic isolate, animals carrying a particular RAB- 5 transgene (red) are compared with siblings lacking the transgene (black). Animals after axotomy at 3 and $6 \mathrm{~h}$ (red) are compared with uncut axons from animals of the same strain (black). Each data point represents one animal; $n \geq 15$. Bars represent the mean of each group with the SD; exact $p$-values from $t$ test.

body in uncut, 3 and $6 \mathrm{~h}$ after axotomy, respectively; $p=0.2675$ when comparing uncut and $3 \mathrm{~h}$ after axotomy, and $p=0.1902$ when comparing uncut and $6 \mathrm{~h}$ after axotomy, $t$ test, $n \geq 8$ ). Together, these data suggest that axotomy has no significant effect on vesicle secretion and that vesicles might not be a necessary requirement for axonal fusion.

Overall, our results are consistent with a model in which these structures are likely generated via accumulation of
EFF-1 at the membrane as a consequence of perturbed RAB- 5 activity, such that EFF-1 is "pinched off" in the form of a vesicle.

\section{RAB-5 controls EFF-1 localization to intracellular compartments}

We next investigated whether RAB-5 also controls EFF-1 localization to intracellular puncta. Given that EFF-1 colocalizes with 
a
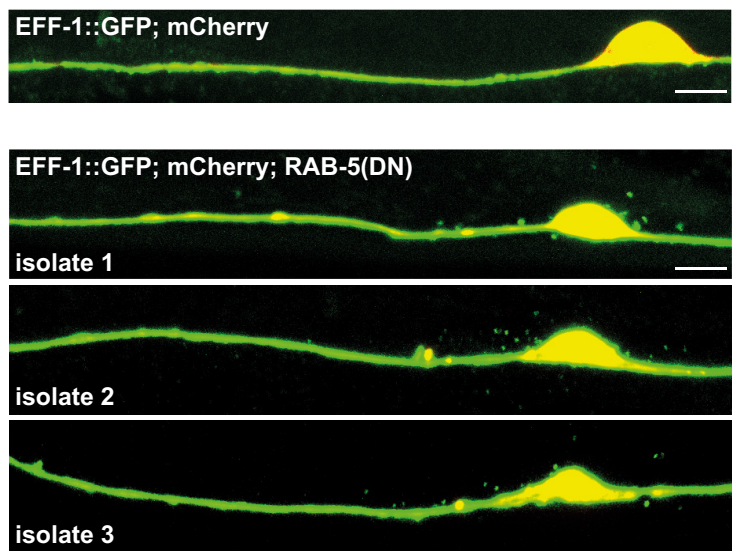

b

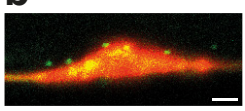

overlay

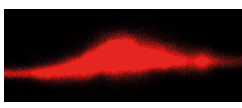

mCherry and myr::mCherry
C

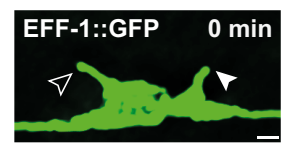

d
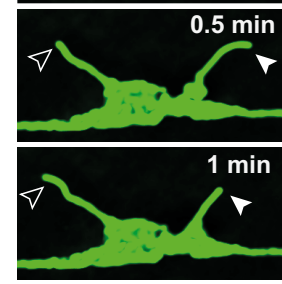

e

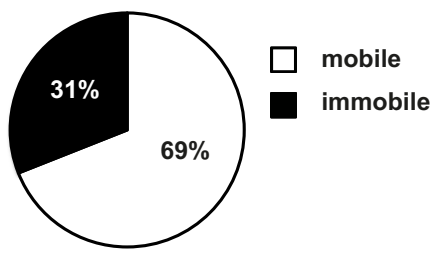

Figure 3. EFF-1 forms extracellular vesicles with perturbed RAB-5 activity. $\boldsymbol{a}$, Representative image of an eff-1 mutant expressing EFF-1::GFP and cytoplasmic mCherry in PLM (no vesicles) compared with three independent transgenic isolates coexpressing RAB- $5(\mathrm{DN})$, in which vesicles are observed around the PLM cell body. Scale bar, $5 \mu \mathrm{m}$. $\boldsymbol{b}$, Single plane confocal image of the PLM cell body in an eff- 1 mutant expressing EFF-1::GFP, RAB-5(DN), and cytoplasmic and membrane-bound mCherry (MYR::mCherry) in PLM. There is no mCherry signal present in the vesicles. Image is demonstrative of 18 animals. Scale bar, $2 \mu$ m. c, Time-lapse images demonstrating dynamic membrane protrusions of EFF-1::GFP in an animal expressing RAB-5(DN). One protrusion is marked with a filled arrowhead, the other with an open arrowhead. Scale bar, $1 \mu \mathrm{m}$. $\boldsymbol{d}$, Time-lapse images demonstrating the budding of a vesicle (arrowhead) over a period of 1 min. Another faint, immobile vesicle can also be seen (asterisk). Scale bar, $1 \mu \mathrm{m}$. $\boldsymbol{e}$, Proportion of mobile versus immobile vesicles, based on 42 vesicles in 24 animals.

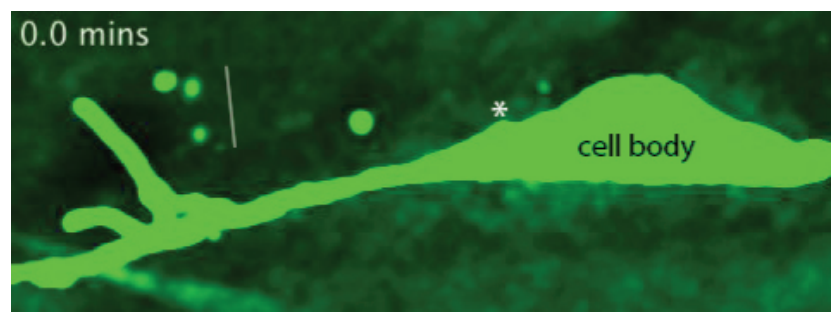

Movie 1. Budding and dynamics of EFF-1 extracellular vesicles. Movie shows the dynamics of EFF-1::GFP vesicles over $3.5 \mathrm{~min}$ in an animal expressing RAB-5(DN). The white bar highlights the position of a group of three vesicles seen to move relative to each other. Also of interest is the budding of a new protrusion from the cell body (asterisk), which may represent a vesicle that is about to separate from the cell.

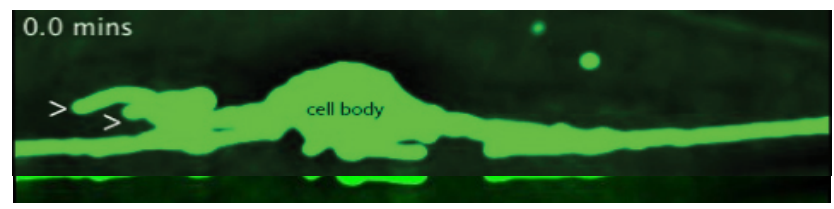

Movie 2. Dynamics of EFF-1 extracellular vesicles and protrusions. Movie shows the dynamics of EFF-1::GFP vesicles and protrusions over 3.5 min in an animal expressing RAB-5(DN). Two dynamic protrusions are noted anterior to the cell body (open arrowheads). Posterior to the cell body, a new vesicle appears at $1 \mathrm{~min}$ (asterisk) and moves relative to two other vesicles.

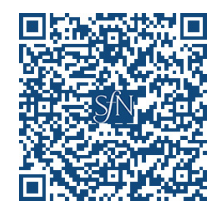

RAB-5 in other cell types (Smurova and Podbilewicz, 2016), we hypothesized that the EFF-1::GFP puncta in PLM neurons represent EFF-1 contained in early endosomes and are formed through RAB-5-mediated endocytosis and endosome fusion. Changes in
RAB-5 activity were therefore expected to alter the morphology of these puncta.

To test this, we expressed the three different versions of RAB-5 described above and characterized the size of the EFF-1::GFP puncta present in the PLM cell body. We used the Squassh imaging tool (ImageJ) (Rizk et al., 2014) to automatically select and measure these puncta. Our results revealed no significant difference in the average size of the puncta detected in RAB-5(DN) cell bodies (Fig. 4a). However, overexpression of RAB-5(WT) caused accumulation of EFF-1 within enlarged intracellular puncta (Fig. 4b). This effect was even more pronounced when RAB-5 activity was increased by RAB-5(CA) (Fig. 4c). This result is consistent with the known role of RAB-5 in endosome fusion and the enlarged endosome phenotype generated by RAB- 5 overactivity (Stenmark et al., 1994; Patton et al., 2005). Importantly, to ascertain the specificity of the phenotype, we also measured the EFF-1 puncta size in animals following axotomy and found no difference at 3 and $6 \mathrm{~h}$ after surgery compared with unoperated animals (Fig. 4d).

To confirm that EFF-1 was accumulating in RAB-5-positive compartments, we performed colocalization studies of EFF-1 with RAB-5 in the context of RAB-5 overexpression. We coexpressed BFP::RAB-5 with EFF-1::GFP in the mechanosensory neurons (Fig. $4 e$ ), which reproduced the enlarged EFF-1 puncta phenotype, leading to a significant increase in average EFF-1 puncta size (Fig. 4e). In these enlarged puncta, the average colocalization of EFF-1 with RAB-5 was $85 \%$ (range $67-98 \% ; n=12$ ). Interestingly, we found that this colocalization became much more variable after axotomy, with an average colocalization with RAB- 5 of only $40 \%$ at both 3 and $6 \mathrm{~h}$ (ranges $0-94 \% ; n=5$ both groups). This is consistent with shifting of EFF-1 to the regenerating growth cone and the cell periphery as part of the regeneration process. Together, these results strongly support the notion that overactivity of RAB-5 results in EFF-1 accumulation in enlarged early endosomes. 
a
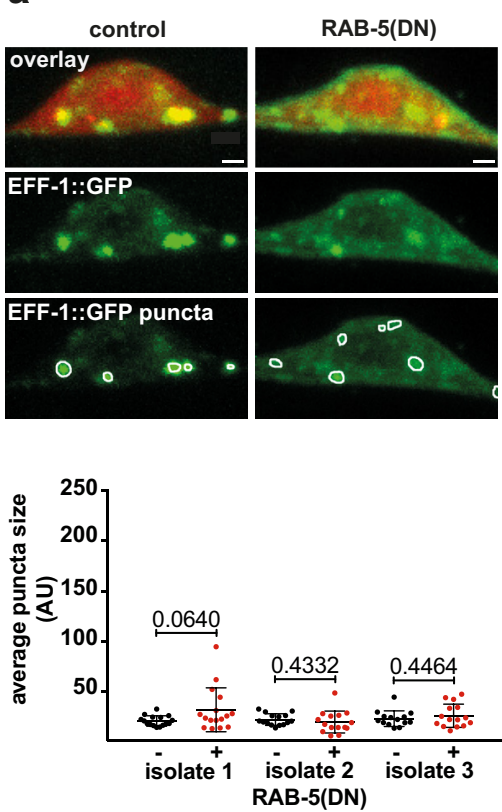

C

control
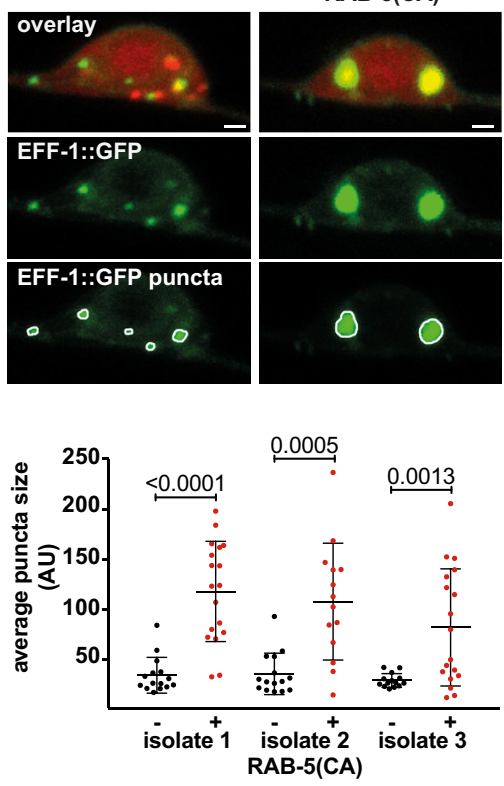

e
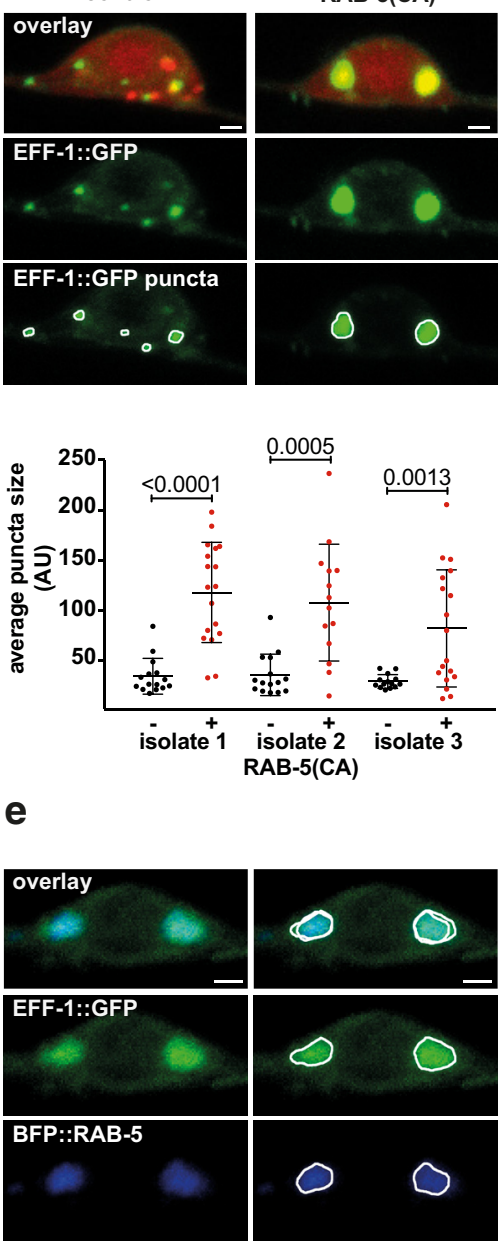

b
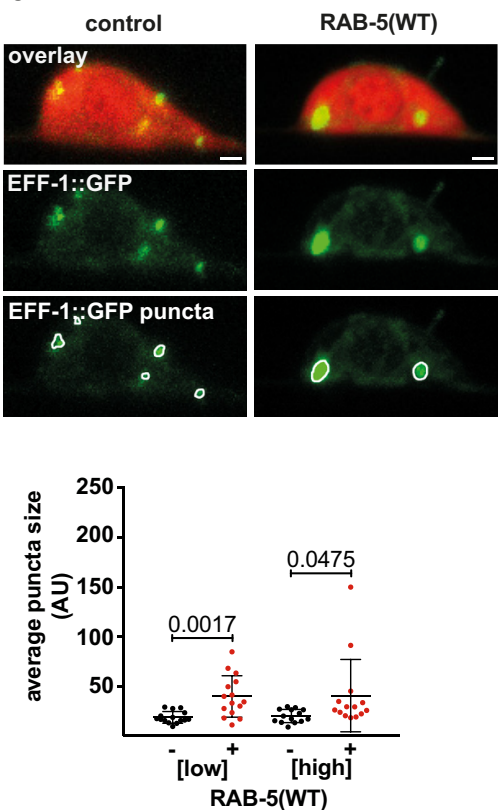

d

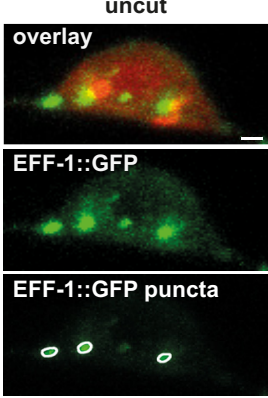

6h post-axotomy
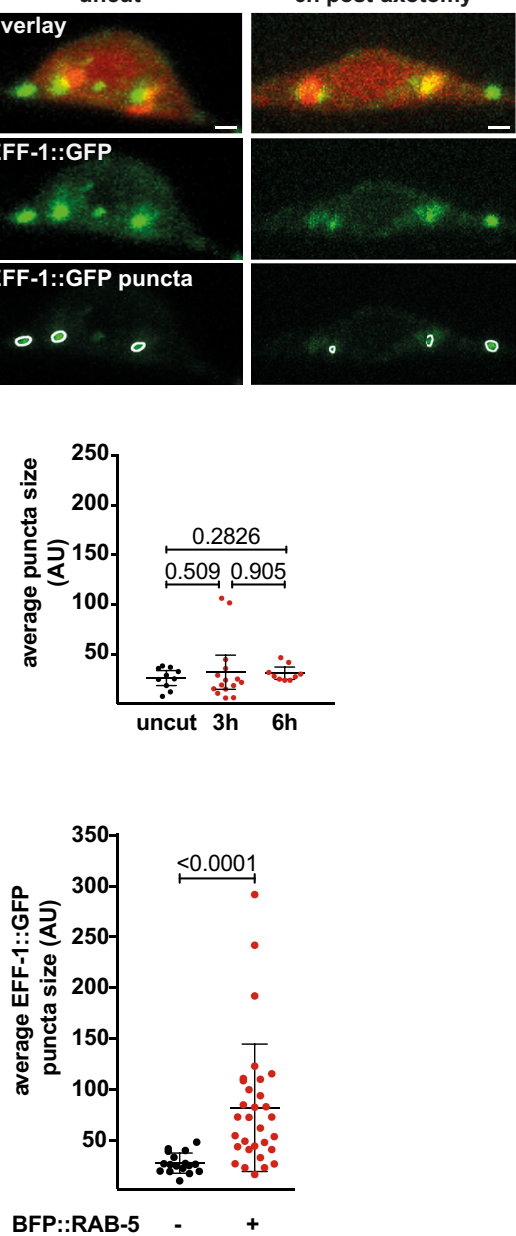

Figure 4. Increased RAB-5 activity causes EFF-1 accumulation in large intracellular puncta, which are RAB-5-positive compartments. $\boldsymbol{a}-\boldsymbol{c}$, Representative maximum projection confocal images of the PLM cell body in eff-1(0k1021) animals expressing RAB-5(DN) (a), RAB-5(WT) (b), or RAB-5(CA) (c) compared with control animals lacking each respective RAB-5 transgene. Displayed for each cell body are the overlay of the red (diffusible mCherry) and green (GFP-tagged EFF-1) channels (top row), the green channel (middle row), and the result of automatic puncta selection using Squassh (bottom row). Scale bars, $1 \mu \mathrm{m}$. Below the confocal images are graphs showing quantification of the average EFF-1::GFP puncta size in independent transgenic isolates
RAB- 5 controls the amount of EFF- 1 in the PLM neuron

Our results indicated that altering RAB-5 activity causes EFF-1 mislocalization to specific subcellular compartments (either the membrane with decreased RAB-5 activity or enlarged endosomes with increased RAB-5 activity). We next investigated whether this mislocalization affected the ability of the neuron to mediate recycling or degradation of the EFF-1 protein. We hypothesized that defects in such processes would result in a buildup of EFF-1::GFP in the neuron. To test this, we measured the average EFF-1::GFP intensity in the PLM axon and cell body of animals expressing RAB-5(DN), RAB5(CA), or RAB-5(WT). We also used an alternative approach to perturb RAB-5 activity using cell-specific RNAi (Esposito et al., 2007), whereby animals expressed sense and antisense rab-5(sas) in the mechanosensory neurons for cell-specific silencing of $r a b-5$. We found that the average EFF-1::GFP intensity in both the axon and cell body was significantly

$\longleftarrow$

expressing each version of RAB-5: RAB-5(DN) (a) RAB-5(WT) (b), or RAB-5(CA) (c). Animals with a given RAB-5 transgene (red) were compared with siblings lacking the transgene (black). A significant increase in average puncta size was found for three independent RAB-5(CA) isolates and for two RAB$5(\mathrm{WT})$ isolates injected at different concentrations $(5 \mathrm{ng} / \mu \mathrm{l}$ and $10 \mathrm{ng} / \mu \mathrm{l})$. Each data point represents one cell body from one animal; $n \geq 14$. Bars represent the mean of each group with the SD; exact $p$-values from $t$ test. AU, Arbitrary units. $d$, Representative maximum projection confocal images of the PLM cell body in an eff-1 (ok1021) animal at $6 \mathrm{~h}$ after axotomy compared with an uncut control animal. As for $\boldsymbol{a}-\boldsymbol{c}$, displayed for each cell body are the overlay of the red (diffusible mCherry) and green (GFP-tagged EFF-1) channels (top row), the green channel (middle row), and the result of automatic puncta selection using Squassh (bottom row). Scale bars, 1 $\mu \mathrm{m}$. Below the confocal images are graphs showing quantification of the average EFF-1::GFP puncta size. Animals at 3 and $6 \mathrm{~h}$ after axotomy (red) are compared with uncut animals (black). No significant difference was noted. Each data point represents one cell body from one animal. Bars represent the mean of each group with the SD. $\boldsymbol{e}$, Representative maximum projection confocal image of the PLM cell body in an animal coexpressing EFF-1::GFP and BFP::RAB-5; the overlay and individual green and blue channels (left column) are matched with the result of automatic puncta selection using Squassh (right column), demonstrating strong colocalization between the EFF-1::GFP and BFP.:RAB-5 puncta. Scale bar, $1 \mu \mathrm{m}$. Next to these confocal images is a graph showing quantification of the average EFF-1::GFP puncta size in a transgenic strain carrying EFF-1::GFP and BFP::RAB-5. A significant increase in EFF1::GFP puncta size occurred when BFP::RAB- 5 was also present $(+)$. The BFP::RAB-5 transgene was injected at the same concentration as the other RAB-5 transgenes. Each data point represents one cell body from one animal; $n \geq 17$. Bars represent the mean of each group with the SD; exact $p$-values from $t$ test. AU, Arbitrary units. 

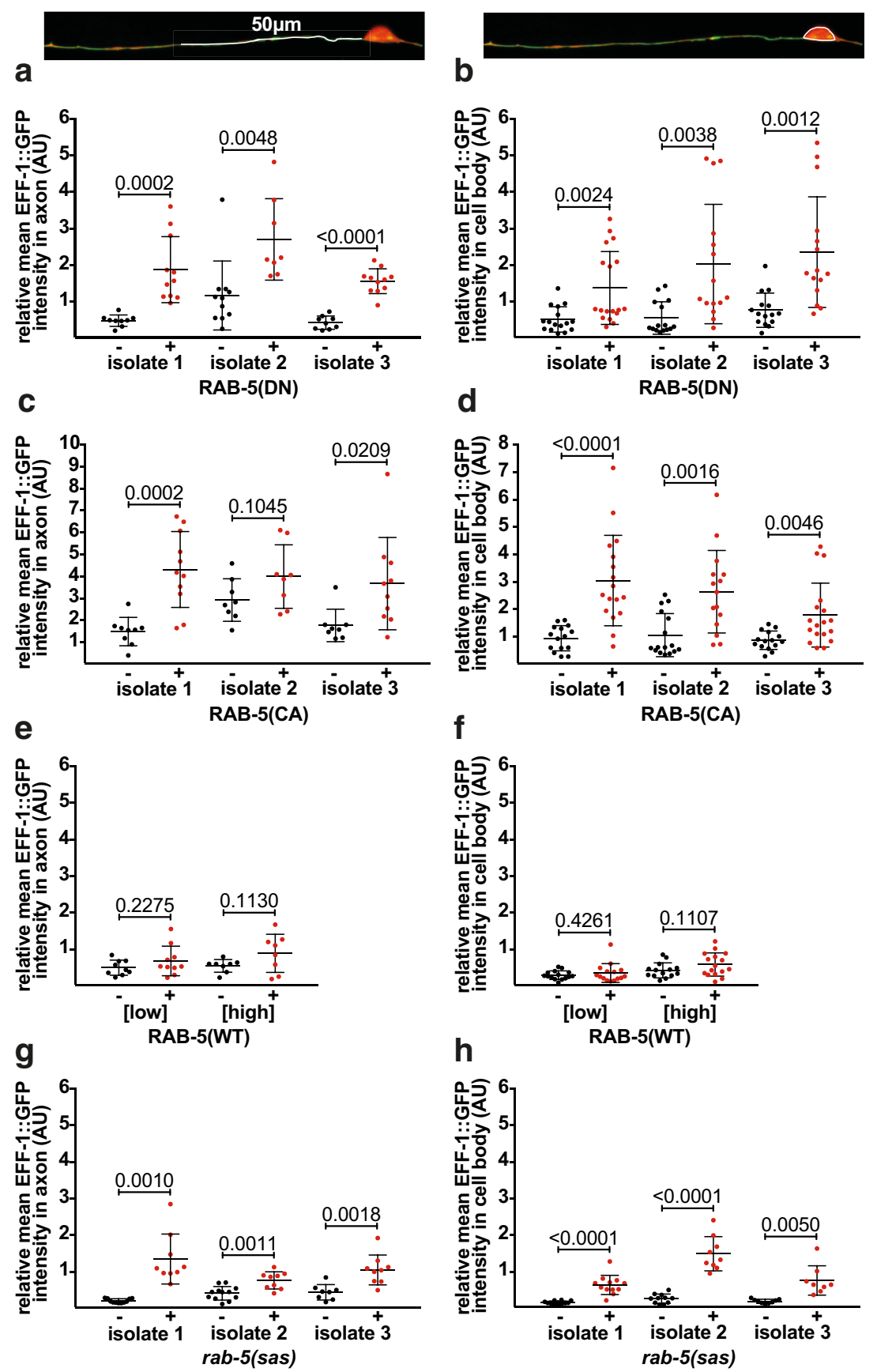

\section{h}
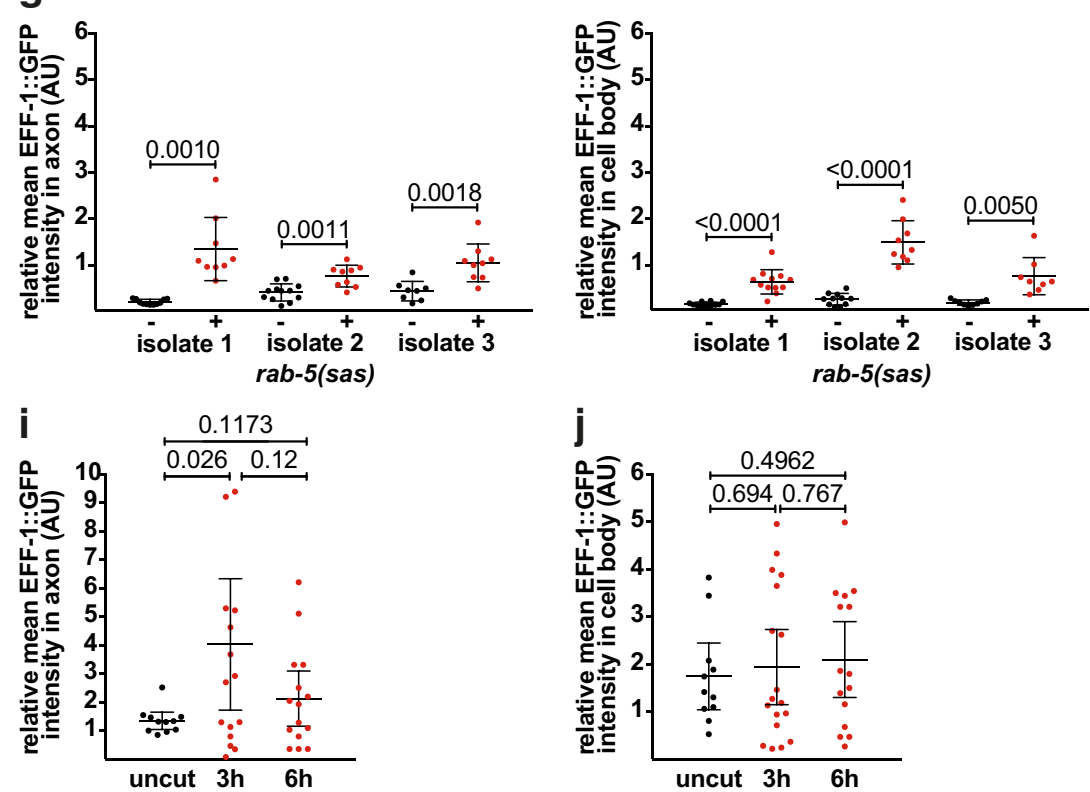

greater in animals expressing either RAB5(DN), RAB-5(CA), or rab-5(sas) (Fig. $5 a-d, g, h)$. This may represent defects in recycling and/or degradation of EFF-1 due to altered RAB- 5 activity. In contrast, the overexpression of RAB-5(WT) had no significant effect on EFF-1::GFP intensity (Fig. 5e,f). This indicates that the RAB$5(\mathrm{WT})$ molecule may be modified by endogenous regulators and thus regulated to minimize changes in EFF-1 levels. Therefore, only unregulated alterations in RAB-5 activity generated with expression of RAB-5(DN), RAB-5(CA), or rab-5(sas) disrupt EFF-1 protein levels.

Moreover, we tested whether axotomy could also alter EFF-1::GFP intensity in PLM at 3 and $6 \mathrm{~h}$ after surgery. We found an increase in axon intensity at $3 \mathrm{~h}$ after surgery (Fig. $5 i$ ), whereas no changes were detected in the cell body (Fig. 5j). This change in mean intensity following axotomy could reflect the change in the EFF-1 localization in the regrowing axon (Neumann et al., 2015).

\section{Other endocytic molecules do not control EFF-1 localization}

A number of molecules function in the endocytosis of cargo from the plasma membrane. We sought to determine whether other endocytic regulators might act in conjunction with RAB-5 to regulate neuronal EFF-1. One important candidate for this role is DYN-1, the C. elegans ortholog of dynamin, because it has been demonstrated to act in this fashion alongside RAB-5 to regulate EFF-1 in the hypodermis (Smurova and Podbilewicz, 2016). We tested whether loss of DYN-1 function, induced using the temperaturesensitive allele dyn-1(ky51), influenced EFF-1::GFP localization in the PLM neurons. Surprisingly, we found no significant effect of DYN-1 on EFF-1::GFP puncta size, membrane localization, or average intensity in the neuron (Fig. $6 a, c, e)$.

We also tested EHS-1, a clathrin adaptor involved in endocytosis that localizes to the plasma membrane. However, lossof-function ehs-1 mutant animals showed no significant change in the same measurements of EFF-1::GFP localization (Fig. 6b,d,f). We believe that this is consistent with studies of ehs-1 in other systems, which indicate that it can act redundantly with other clathrin adaptors (Mayers et al., 2013). Therefore, identifying its potential role in this process requires further investigation.

From early endosomes, the endocytic pathway allows for transport of cargo to intensity in the axon (left column) and cell body (right column) of eff-1(0k1021) animals coexpressing EFF-1 *: GFP with different RAB-5 transgenes or animals after axotomy. Each mean GFP measurement is expressed relative to the mean mCherry intensity in the same region (see Materials and Methods). This intensity was significantly increased with expression of RAB-5(DN) $(\boldsymbol{a}, \boldsymbol{b})$, RAB-5(CA) (c, d ), or rab-5(sas) $(\boldsymbol{g}, \boldsymbol{h})$ and in the axon at $3 \mathrm{~h}$ after axotomy (i). No significant difference was found with expression of RAB-5(WT) $(\boldsymbol{e}, \boldsymbol{f})$, nor in the cell body after axotomy $(\boldsymbol{j})$. Each point represents the mean for a single axon or cell body; $n \geq 8$. Bars represent the mean of each group with the SD; exact $p$-values from $t$ test. AU, Arbitrary units. 

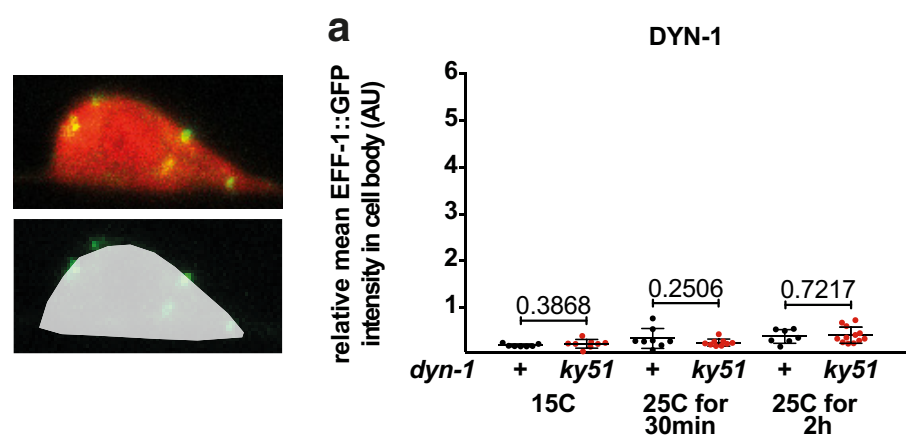

C
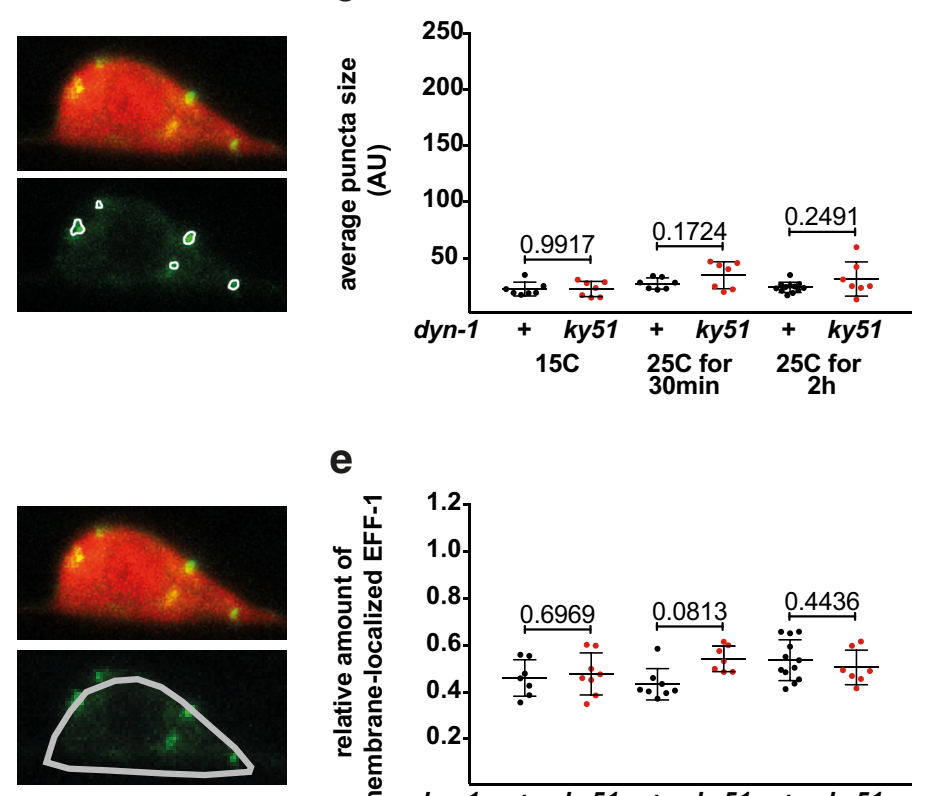

e

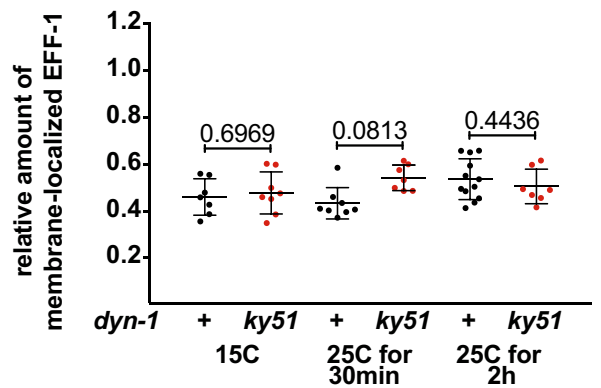

b EHS-1

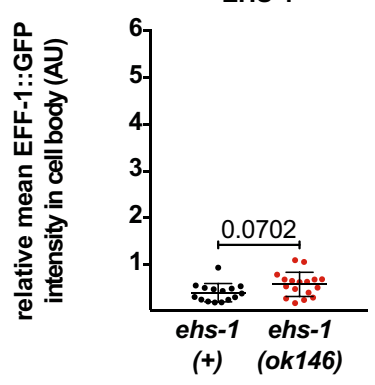

d

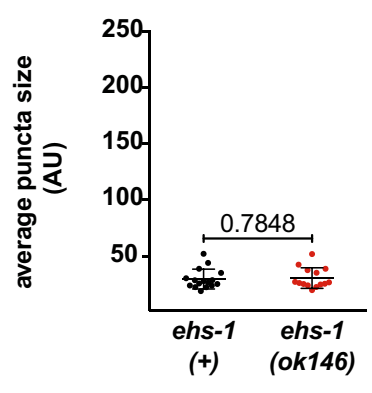

f

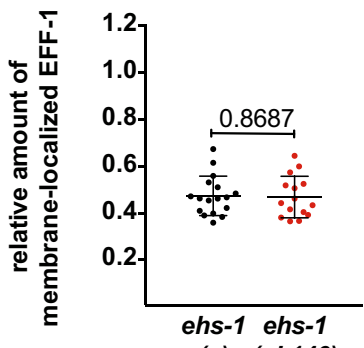

(+) (ok146)

Figure 6. The endocytic molecules DYN-1 and EHS-1 have no effect on EFF-1 localization in PLM. Analyses of EFF-1::GFP localization in the presence of the temperature-sensitive allele dyn-1(ky51) $(\boldsymbol{a}, \boldsymbol{c}, \boldsymbol{e})$ or the loss-of-function allele ehs-1(ok146) (b, $\boldsymbol{d}, \boldsymbol{f})$. No significant difference was found in EFF-1::GFP intensity (top row), the average EFF-1::GFP puncta size (middle row), or the amount of EFF- 1 at the membrane (bottom row). To test dyn-1(ky51), animals maintained at the permissive temperature $\left(15^{\circ} \mathrm{C}\right.$ ) were compared with those shifted to the restrictive temperature $\left(25^{\circ} \mathrm{C}\right)$ for $30 \mathrm{~min}$ or $2 \mathrm{~h}$. Each point represents the mean for a single cell body; $n \geq 7$. Bars represent the mean with the SD; exact $p$-values from $t$ test. AU, Arbitrary units.

downstream compartments, potentially for membrane recycling or degradation. We therefore investigated whether molecules localizing to these compartments also participated in EFF-1 regulation. A number of RAB proteins presented good candidates; these included RAB-7, which functions in endosome-tolysosome trafficking, RAB-10, an endocytic recycling regulator that localizes to endosomes and Golgi, and RAB-11, which controls transport between recycling endosomes and the plasma membrane. We perturbed the function of these $r a b$ genes in the PLM neurons either through expression of dominant-negative versions or by using loss-of-function alleles. However, our results indicated that these RAB molecules were not involved in EFF-1 localization in the PLM neurons. Neither expression of dominant-negative RAB-7 or RAB-11 nor the presence of the rab-10(dx2) loss-of-function allele significantly altered our measurements of EFF-1::GFP intensity or localization (Fig. 7). Overall, these results support the specificity of our findings with RAB-5 and suggest that this molecule is a key regulator of neuronal EFF-1 and has the ability to regulate axonal fusion.

\section{Discussion}

Our data demonstrate that perturbing RAB-5 activity in PLM neurons has a clear functional effect on axonal repair. Specifically, the presence of RAB-5(DN) increased the capacity for EFF-1 to mediate axonal fusion because it phenocopied EFF-1 overexpression by rescuing the psr-1 axonal fusion defect. By visualizing EFF-1, we determined that this change in activity reflected mobilization of EFF-1 to the plasma membrane, which also occurs with reduced RAB-5 function in the C. elegans hypodermis (Smurova and Podbilewicz, 2016).

Interestingly, the presence of RAB-5(CA) did not produce the opposite phenotype because it did not significantly reduce axonal fusion efficiency (Fig. 1e) or remove greater amounts of EFF-1 from the neuronal membrane (Fig. 2). It is possible that there was insufficient overactivity of RAB-5 in the strains tested to fully remove EFF-1 from the membrane or that only a small number of EFF-1 molecules is required at the membrane for fusion and sufficient amounts were present even in the presence of RAB5(CA). Alternatively, it is possible that a RAB-5-independent 

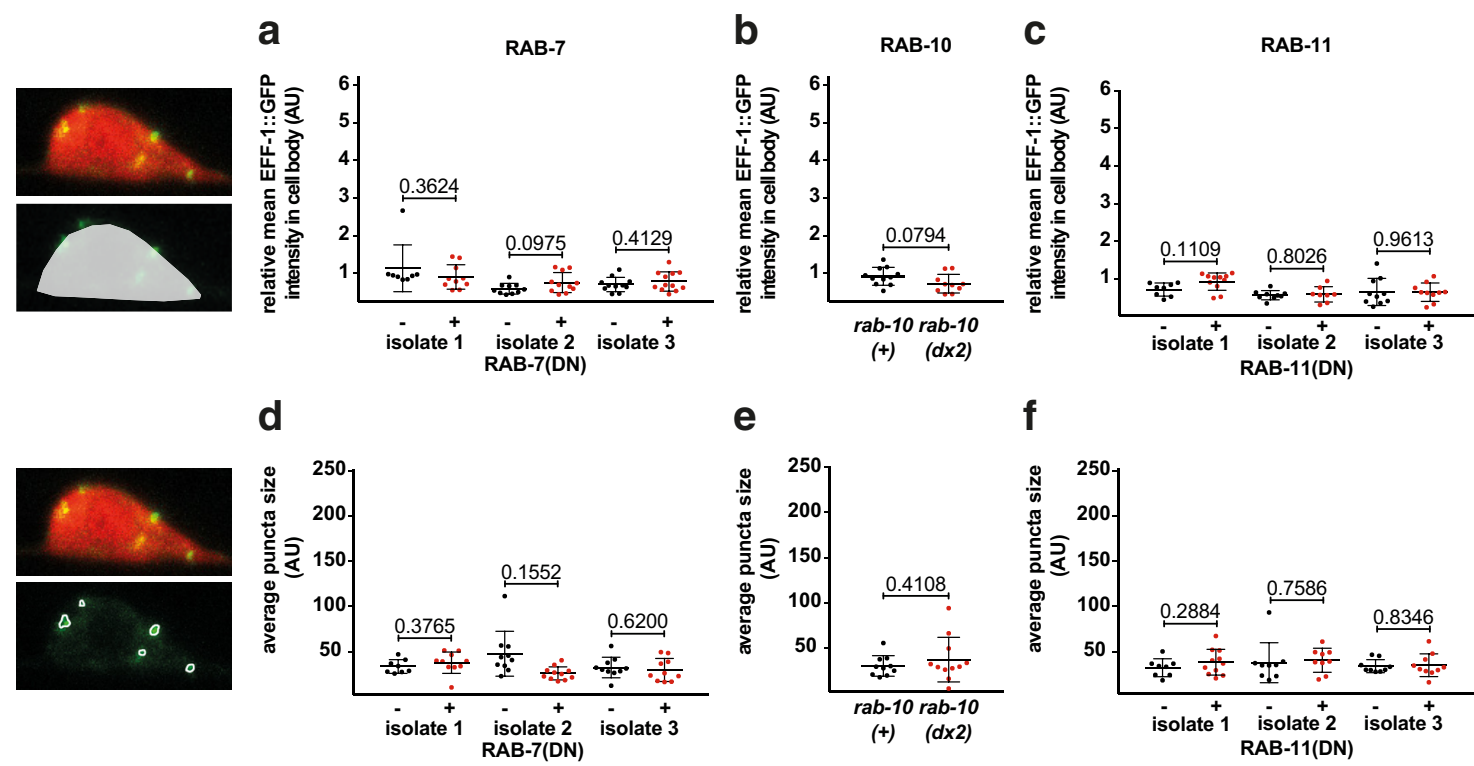

g
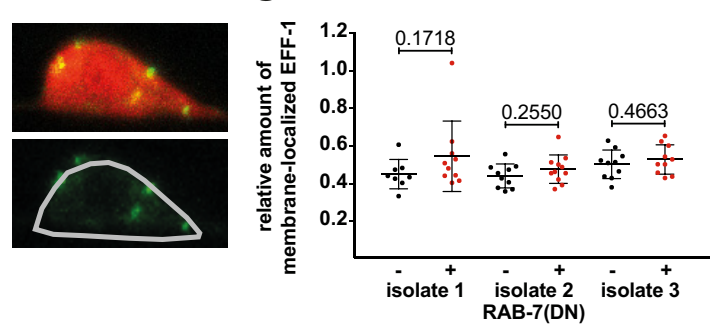

h

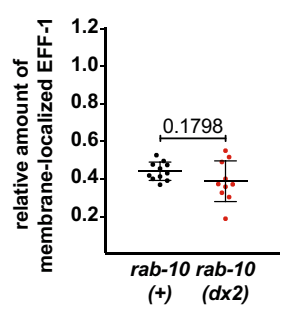

i

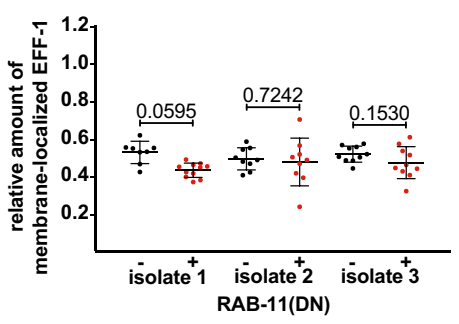

Figure 7. Other RAB molecules have no effect on EFF-1 localization in PLM. Analyses of EFF-1::GFP localization in independent transgenic isolates coexpressing RAB-7(DN) (a, $\boldsymbol{d}, \boldsymbol{g})$, rab-10(dx2) $(\boldsymbol{b}, \boldsymbol{e}, \boldsymbol{h})$, or RAB-11(DN) $(\boldsymbol{c}, \boldsymbol{f}, \boldsymbol{i})$. For each isolate tested, no significant difference was found in the relative mean EFF-1::GFP intensity (top row), average EFF-1::GFP puncta size (middle row), or amount of EFF-1 at the membrane (bottom row). Animals with the altered rab gene of interest (red) are compared with siblings WT for that rab gene (black). Each point represents the mean for a single cell body; $n \geq 8$; bars represent the mean of each group with the SD; exact $p$-values from $t$ test. AU, Arbitrary units.

mechanism exists for the mobilization of EFF-1 to the membrane after injury. Recruitment of EFF-1 to fusion sites in larval hypodermal cells has been shown to be mediated at least in part by the actin regulator VAB-10 (Yang et al., 2017), although other pathways may also exist. The molecular cascades that are activated in regenerating axons have been well characterized (El Bejjani and Hammarlund, 2012; Hammarlund and Jin, 2014; Byrne and Hammarlund, 2017) and it is plausible that some of these molecules play a currently uncharacterized role in EFF-1 recruitment.

However, overactivity of RAB-5 did lead to accumulation of EFF-1 in enlarged RAB-5-positive compartments (Fig. 4c,d). Previous studies have documented $45-69 \%$ colocalization of endogenous EFF-1 with RAB-5 in hypodermal cells (Smurova and Podbilewicz, 2016). Because our model visualizes overexpression of RAB-5, our results are not directly comparable and we are unable to conclude to what extent EFF-1 colocalizes with RAB5 (WT) levels in the neuron. However, our findings are consistent with a role for RAB-5 in determining steady-state EFF-1 localization to early endosomes.

Altering RAB-5 activity also increased the intracellular amount of EFF-1, as reflected by increases in EFF-1::GFP intensity. This occurred in both the axon and the cell body, indicating that it likely represents protein accumulation rather than an axonal transport defect. It was also observed specifically in the presence of RAB- 5 molecules with locked activity states, which were associated with abnormal accumulation of EFF-1 either at the membrane or in early endosomes. Although no molecules have yet been confirmed as regulators of EFF-1 degradation, there is some evidence that the vacuolar $\mathrm{H}+$-ATPase complex may perform this role in the C. elegans hypodermis. The vha-17 gene, which encodes a subunit of this complex, has been found to suppress EFF-1-mediated hypodermal fusion (Kontani et al., 2005). It does not appear to influence EFF-1 membrane localization (Smurova and Podbilewicz, 2016), but has been speculated to act in an EFF-1 degradation pathway (Kontani et al., 2005), although this has not been specifically tested. In the case of RAB-5, there is good precedence for this molecule functioning in protein degradation and homeostasis. In the C. elegans embryo, it is required for both endocytosis and degradation of the C. elegans caveolin CAV-1 (Sato et al., 2006). In HeLa cells, either increasing or decreasing RAB-5 activity (achieved indirectly using a regulator of RAB-5) was found to perturb endocytic trafficking and lead to cargo build-up in specific compartments (Haas et al., 2005). We propose that the accumulation of EFF-1, either at the membrane with RAB-5(DN) or in abnormally enlarged endosomes with RAB$5(\mathrm{CA})$, occurred due to defects in the trafficking of EFF-1 from these structures. Interestingly, greater intracellular levels of EFF-1 per se did not guarantee an improvement in the efficiency of axonal fusion. Rather, our results indicate that EFF-1 must specifically accumulate at the membrane to improve fusion capacity.

Our findings are consistent with a model in which RAB-5 mediates the endocytosis of EFF-1 from the PLM cell membrane, similar to that described in the hypodermis (Smurova and Podbilewicz, 2016). In our model (Fig. 8), EFF-1 is transiently in- 

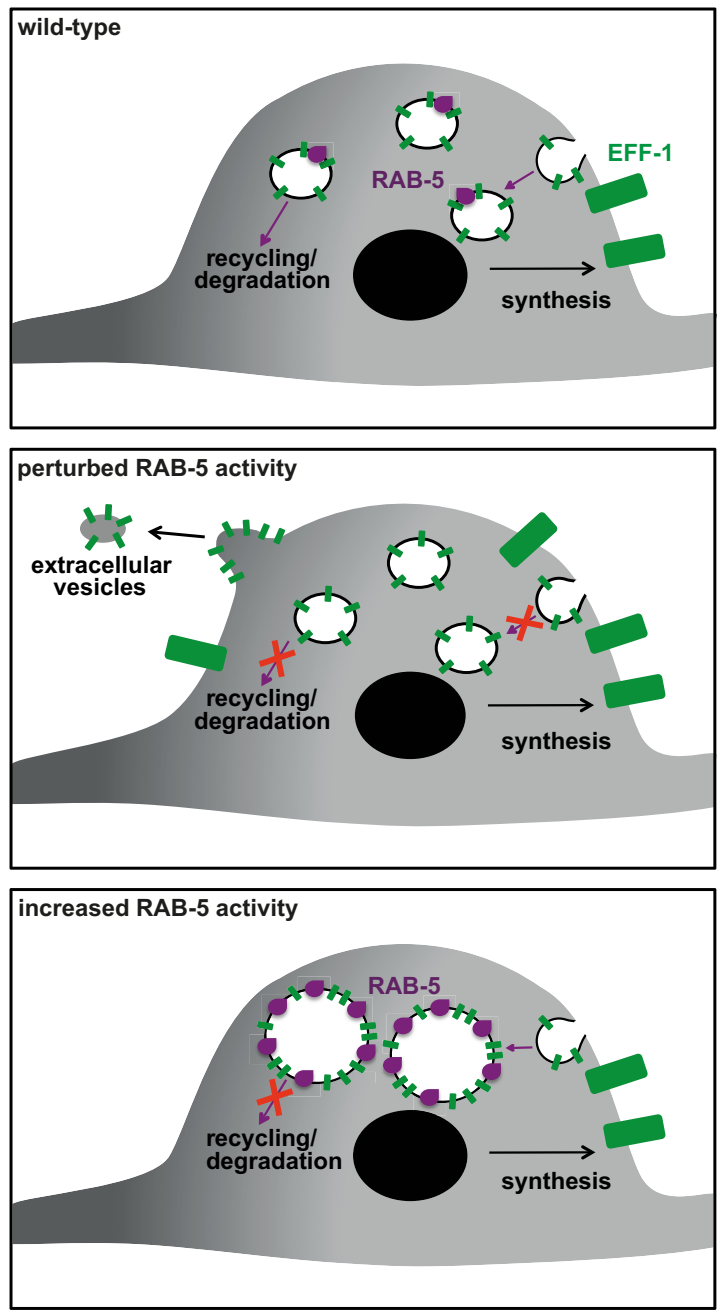

Figure 8. Model of RAB-5 regulation of EFF-1 in PLM. In the WT scenario, EFF-1 (green) is transiently inserted into the membrane following synthesis. Active RAB-5 (purple) functions in the removal of EFF-1 into early endosomes. This in turn is required for EFF-1 trafficking into downstream compartments for recycling/degradation. When RAB-5 function is perturbed, EFF-1 is no longer removed from the membrane and subsequent accumulation at the membrane results in membranous protrusions and budding of extracellular vesicles. There is an additional failure of trafficking into recycling/degradation pathways. With increased RAB-5 activity, EFF-1 instead accumulates in enlarged early endosomes. This endosomal enlargement also leads to a defect in downstream trafficking of EFF-1 for recycling/degradation.

serted into the plasma membrane following synthesis. Active RAB-5 is responsible for subsequent transport of this EFF-1 into early endosomes, where EFF-1 largely resides in the steady state. Decreasing RAB-5 activity allows EFF-1 accumulation at the membrane, whereas overactivity of RAB-5 leads to accumulation in early endosomes. We also propose that, in neurons, RAB-5-mediated endocytosis occurs upstream of pathways for EFF-1 recycling/degradation. Because altering RAB-5 activity sequesters EFF-1 in specific compartments, it prevents downstream trafficking and creates a build-up of EFF-1 in the neuron. It remains to be determined whether RAB-5 also regulates EFF-1 in the context of phagosome sealing, as described for the removal of debris from the tail spike cell in C. elegans (Ghose et al., 2018).

A particularly fascinating aspect of our study was the generation of extracellular EFF-1::GFP-positive vesicles when RAB-5 activity was perturbed. Extracellular vesicles were observed incidentally in the earliest studies of EFF-1-mediated cell-cell fusion using electron microscopy (Mohler et al., 1998). Vesicles that specifically contain fusogens have now been documented in vitro (Avinoam et al., 2011; Zeev-Ben-Mordehai et al., 2014) and more recently in vivo in C. elegans (Oren-Suissa et al., 2017), but their exact characteristics and functionality have yet to be elucidated. The absence of both membrane and cytoplasmic markers in the EFF-1::GFP vesicles suggests that these vesicles may exclude some membrane proteins, such as fluorophores, or contain insufficient amounts for visualization with confocal microscopy. However, it appears unlikely that they contain purely EFF-1::GFP because fusogen-containing vesicles in vitro have been shown to contain other proteins (Zeev-Ben-Mordehai et al., 2014), as well as membrane proteins in some cases (Avinoam et al., 2011). We therefore postulate that these vesicles may be selective in their uptake of membrane proteins and that their content could differ from the original composition of the PLM plasma membrane. Given that extracellular vesicles are known to deliver cargo in diverse systems (Tkach and Théry, 2016), fusogen-containing vesicles represent an attractive vehicle for imparting fusion competence to surrounding tissues and potentially to mammalian neurons, as postulated previously (Oren-Suissa et al., 2017). The evidence that these vesicles may be selective and largely contain fusogen suggests that they could be very efficient in delivering fusogenicity, although whether the EFF-1::GFP vesicles have fusogenic activity is currently unclear.

Another matter of speculation is the mechanism through which the EFF-1::GFP vesicles are generated. It remains to be determined whether they are passively extruded or are instead actively secreted. Given that they occur in the presence of increased intracellular and membranous EFF-1, it is plausible that they are created through excessive build-up of EFF-1 at the membrane. With its known function in membrane sculpting (OrenSuissa et al., 2010), EFF-1 could potentially "pinch off" a section of membrane. This may be similar to the recently demonstrated function of AFF-1, another C. elegans fusogen, in mediating endocytic fission events as part of development of the excretory duct (Soulavie et al., 2018). Consistent with this, the vesicles localize in the vicinity of the PLM cell body and it may be that the higher volume-to-surface-area ratio in this part of the neuron allows for sufficient build-up of EFF-1::GFP behind the membrane. In support of this, most protrusions, and the observed events of vesicle budding, originated from the cell body.

$\mathrm{RAB}$ proteins have well established roles in intracellular trafficking and we tested a suite of molecules other than RAB-5 that could potentially regulate such transport of EFF-1 in PLM. As reported for the hypodermis (Smurova and Podbilewicz, 2016), RAB-7, RAB-10, and RAB-11 did not influence EFF-1 localization. Similarly, we did not find a role for DYN-1, although this molecule has been demonstrated to negatively regulate EFF-1 and cell-cell fusion in the hypodermis (Smurova and Podbilewicz, 2016). However, the literature on dynamin suggests that it can have varying roles in fusion events in different systems related to its multiple functions in endocytosis as well as actin cytoskeletal rearrangements. In some mammalian cell-cell fusions, including in osteoclasts and myoblasts, dynamin activity appears to instead promote fusion (Leikina et al., 2013; Shin et al., 2014; Verma et al., 2014). Our result is consistent with an alternative role for DYN-1 in the neuron, potentially in endocytic regulation of molecules other than EFF-1.

In summary, our study identifies RAB-5 as a key regulator of EFF-1 in the nervous system. By controlling the levels of EFF-1 in endosomal compartments, RAB-5 modulates the amount of this protein available on the membrane, thereby regulating its fuso- 
genic capacity. Therefore, manipulating the activity of RAB-5 activity provides a means to promote highly efficient neuronal repair through axonal fusion. Although EFF-1 does not have a direct human homolog, several mammalian fusogens have been identified, including human syncytin-1 and syncytin-2 (Mi et al., 2000; Blaise et al., 2003). Very little is known about the regulation of these fusogens in vivo, but it is tempting to speculate that these molecules, similarly to EFF-1, might be trafficked to the membrane for fusion and then recycled using the endocytic pathway. We therefore suggest that RAB-5 could play a role in the regulation of fusogen activity or localization, making it a target for the modulation of fusogenic activity in mammalian cells.

\section{References}

Abay ZC, Wong MY, Teoh JS, Vijayaraghavan T, Hilliard MA, Neumann B (2017) Phosphatidylserine save-me signals drive functional recovery of severed axons in Caenorhabditis elegans. Proc Natl Acad Sci U S A 114:E10196-E10205.

Avinoam O, Fridman K, Valansi C, Abutbul I, Zeev-Ben-Mordehai T, Maurer UE, Sapir A, Danino D, Grünewald K, White JM, Podbilewicz B (2011) Conserved eukaryotic fusogens can fuse viral envelopes to cells. Science 332:589-592.

Basu A, Dey S, Puri D, Das Saha N, Sabharwal V, Thyagarajan P, Srivastava P, Koushika SP, Ghosh-Roy A (2017) let-7 miRNA controls CED-7 homotypic adhesion and EFF-1-mediated axonal self-fusion to restore touch sensation following injury. Proc Natl Acad Sci U S A 114:E10206E10215.

Bedi SS, Glanzman DL (2001) Axonal rejoining inhibits injury-induced long-term changes in Aplysia sensory neurons in vitro. J Neurosci 21: 9667-9677.

Birse SC, Bittner GD (1976) Regeneration of giant axons in earthworms. Brain Res 113:575-581.

Blaise S, de Parseval N, Bénit L, Heidmann T (2003) Genomewide screening for fusogenic human endogenous retrovirus envelopes identifies syncytin 2, a gene conserved on primate evolution. Proc Natl Acad Sci U S A 100: 13013-13018.

Brenner S (1974) The genetics of Caenorhabditis elegans. Genetics 77:71-94.

Bucci C, Parton RG, Mather IH, Stunnenberg H, Simons K, Hoflack B, Zerial M (1992) The small GTPase rab5 functions as a regulatory factor in the early endocytic pathway. Cell 70:715-728.

Byrne AB, Hammarlund M (2017) Axon regeneration in C. elegans: worming our way to mechanisms of axon regeneration. Exp Neurol 287:300309.

del Campo JJ, Opoku-Serebuoh E, Isaacson AB, Scranton VL, Tucker M, Han M, Mohler WA (2005) Fusogenic activity of EFF-1 is regulated via dynamic localization in fusing somatic cells of C. elegans. Curr Biol 15:413423.

Deriemer SA, Elliott EJ, Macagno ER, Muller KJ (1983) Morphological evidence that regenerating axons can fuse with severed axon segments. Brain Res 272:157-161.

El Bejjani R, Hammarlund M (2012) Neural regeneration in Caenorhabditis elegans. Annu Rev Genet 46:499-513.

Esposito G, Di Schiavi E, Bergamasco C, Bazzicalupo P (2007) Efficient and cell specific knock-down of gene function in targeted C. elegans neurons. Gene 395:170-176.

Ghose P, Rashid A, Insley P, Trivedi M, Shah P, Singhal A, Lu Y, Bao Z, Shaham S (2018) EFF-1 fusogen promotes phagosome sealing during cell process clearance in Caenorhabditis elegans. Nat Cell Biol 20:393-399.

Ghosh-Roy A, Wu Z, Goncharov A, Jin Y, Chisholm AD (2010) Calcium and cyclic AMP promote axonal regeneration in Caenorhabditis elegans and require DLK-1 kinase. J Neurosci 30:3175-3183.

Gorvel JP, Chavrier P, Zerial M, Gruenberg J (1991) Rab5 controls early endosome fusion in vitro. Cell 64:915-925.

Haas AK, Fuchs E, Kopajtich R, Barr FA (2005) A GTPase-activating protein controls Rab5 function in endocytic trafficking. Nat Cell Biol 7:887-893.

Hammarlund M, Jin Y (2014) Axon regeneration in C. elegans. Curr Opin Neurobiol 27:199-207.

Hoy RR, Bittner GD, Kennedy D (1967) Regeneration in crustacean motoneurons: evidence for axonal fusion. Science 156:251-252.

Hutagalung AH, Novick PJ (2011) Role of RAB GTPases in membrane traffic and cell physiology. Physiol Rev 91:119-149.
Kontani K, Moskowitz IP, Rothman JH (2005) Repression of cell-cell fusion by components of the C. elegans vacuolar ATPase complex. Dev Cell 8:787-794.

Leikina E, Melikov K, Sanyal S, Verma SK, Eun B, Gebert C, Pfeifer K, Lizunov VA, Kozlov MM, Chernomordik LV (2013) Extracellular annexins and dynamin are important for sequential steps in myoblast fusion. J Cell Biol 200:109-123.

Liu QA, Hengartner MO (1998) Candidate adaptor protein CED-6 promotes the engulfment of apoptotic cells in C. elegans. Cell 93:961-972.

Macagno ER, Muller KJ, DeRiemer SA (1985) Regeneration of axons and synaptic connections by touch sensory neurons in the leech central nervous system. J Neurosci 5:2510-2521.

Mayers JR, Wang L, Pramanik J, Johnson A, Sarkeshik A, Wang Y, Saengsawang W, Yates JR $3^{\text {rd }}$, Audhya A (2013) Regulation of ubiquitindependent cargo sorting by multiple endocytic adaptors at the plasma membrane. Proc Natl Acad Sci U S A 110:11857-11862.

Mello C, Fire A (1995) DNA transformation. Methods Cell Biol 48:451-482.

Mi S, Lee X, Li X, Veldman GM, Finnerty H, Racie L, LaVallie E, Tang XY, Edouard P, Howes S, Keith JC Jr, McCoy JM (2000) Syncytin is a captive retroviral envelope protein involved in human placental morphogenesis. Nature 403:785-789.

Mohler WA, Simske JS, Williams-Masson EM, Hardin JD, White JG (1998) Dynamics and ultrastructure of developmental cell fusions in the Caenorhabditis elegans hypodermis. Curr Biol 8:1087-1090.

Mohler WA, Shemer G, del Campo JJ, Valansi C, Opoku-Serebuoh E, Scranton V, Assaf N, White JG, Podbilewicz B (2002) The type I membrane protein EFF-1 is essential for developmental cell fusion. Dev Cell 2:355362.

Neumann B, Nguyen KC, Hall DH, Ben-Yakar A, Hilliard MA (2011) Axonal regeneration proceeds through specific axonal fusion in transected C. elegans neurons. Dev Dyn 240:1365-1372.

Neumann B, Coakley S, Giordano-Santini R, Linton C, Lee ES, Nakagawa A, Xue D, Hilliard MA (2015) EFF-1-mediated regenerative axonal fusion requires components of the apoptotic pathway. Nature 517:219-222.

Oren-Suissa M, Hall DH, Treinin M, Shemer G, Podbilewicz B (2010) The fusogen EFF-1 controls sculpting of mechanosensory dendrites. Science 328:1285-1288.

Oren-Suissa M, Gattegno T, Kravtsov V, Podbilewicz B (2017) Extrinsic repair of injured dendrites as a paradigm for regeneration by fusion in Caenorhabditis elegans. Genetics 206:215-230.

Patton A, Knuth S, Schaheen B, Dang H, Greenwald I, Fares H (2005) Endocytosis function of a ligand-gated ion channel homolog in Caenorhabditis elegans. Curr Biol 15:1045-1050.

Pérez-Vargas J, Krey T, Valansi C, Avinoam O, Haouz A, Jamin M, RavehBarak H, Podbilewicz B, Rey FA (2014) Structural basis of eukaryotic cell-cell fusion. Cell 157:407-419.

Rizk A, Paul G, Incardona P, Bugarski M, Mansouri M, Niemann A, Ziegler U, Berger P, Sbalzarini IF (2014) Segmentation and quantification of subcellular structures in fluorescence microscopy images using squassh. Nat Protoc 9:586-596.

Sambrook J, Fritsch EF, Maniatis T (1989) Molecular cloning: a laboratory manual. Cold Spring Harbor, NY: Cold Spring Harbor Laboratory.

Sato K, Sato M, Audhya A, Oegema K, Schweinsberg P, Grant BD (2006) Dynamic regulation of caveolin-1 trafficking in the germline and embryo of Caenorhabditis elegans. Mol Biol Cell 17:3085-3094.

Shin NY, Choi H, Neff L, Wu Y, Saito H, Ferguson SM, De Camilli P, Baron R (2014) Dynamin and endocytosis are required for the fusion of osteoclasts and myoblasts. J Cell Biol 207:73-89.

Smurova K, Podbilewicz B (2016) RAB-5- and DYNAMIN-1-mediated endocytosis of EFF-1 fusogen controls cell-cell fusion. Cell Rep 14:15171527.

Soulavie F, Hall DH, Sundaram MV (2018) The AFF-1 exoplasmic fusogen is required for endocytic scission and seamless tube elongation. Nat Commun 9:1741.

Stenmark H, Parton RG, Steele-Mortimer O, Lütcke A, Gruenberg J, Zerial M (1994) Inhibition of rab5 GTPase activity stimulates membrane fusion in endocytosis. EMBO J 13:1287-1296.

Tkach M, Théry C (2016) Communication by extracellular vesicles: where we are and where we need to go. Cell 164:1226-1232. 
Verma SK, Leikina E, Melikov K, Chernomordik LV (2014) Late stages of the synchronized macrophage fusion in osteoclast formation depend on dynamin. Biochem J 464:293-300.

Wang X, Wu YC, Fadok VA, Lee MC, Gengyo-Ando K, Cheng LC, Ledwich D, Hsu PK, Chen JY, Chou BK, Henson P, Mitani S, Xue D (2003) Cell corpse engulfment mediated by $C$. elegans phosphatidylserine receptor through CED-5 and CED-12. Science 302:1563-1566.

Wang X, Li W, Zhao D, Liu B, Shi Y, Chen B, Yang H, Guo P, Geng X, Shang Z, Peden E, Kage-Nakadai E, Mitani S, Xue D (2010) Caenorhabditis elegans transthyretin-like protein TTR-52 mediates recognition of apoptotic cells by the CED-1 phagocyte receptor. Nat Cell Biol 12:655-664.

Wu YC, Horvitz HR (1998) The C. elegans cell corpse engulfment gene ced-7 encodes a protein similar to ABC transporters. Cell 93:951-960.

Yang Y, Zhang Y, Li WJ, Jiang Y, Zhu Z, Hu H, Li W, Wu JW, Wang ZX, Dong MQ, Huang S, Ou G (2017) Spectraplakin induces positive feedback between fusogens and the actin cytoskeleton to promote cell-cell fusion. Dev Cell 41:107-120.e4.

Zeev-Ben-Mordehai T, Vasishta D, Siebert CA, Grünewald K (2014) The full-length cell-cell fusogen EFF-1 is monomeric and upright on the membrane. Nat Commun 5:3912.

Zeigerer A, Gilleron J, Bogorad RL, Marsico G, Nonaka H, Seifert S, EpsteinBarash H, Kuchimanchi S, Peng CG, Ruda VM, Del Conte-Zerial P, Hengstler JG, Kalaidzidis Y, Koteliansky V, Zerial M (2012) Rab5 is necessary for the biogenesis of the endolysosomal system in vivo. Nature 485:465-470.

Zerial M, McBride H (2001) Rab proteins as membrane organisers. Nat Rev Mol Cell Biol 2:107-117.

Zhang Y, Wang H, Kage-Nakadai E, Mitani S, Wang X (2012) C. elegans secreted lipid-binding protein NRF-5 mediates PS appearance on phagocytes for cell corpse engulfment. Curr Biol 22:1276-1284. 